\title{
Giant Thermoelectric Figure of Merit in Fluorine Doped Single Walled Carbon Nanotube
}

\author{
D. Sakyi-Arthur ${ }^{\mathrm{a}}$, S. Y. Mensah ${ }^{\mathrm{a}}$, N. G. Mensah ${ }^{\mathrm{b}}$, K.W. Adu ${ }^{\mathrm{c}, \mathrm{d}, *}$, K. A. \\ Dompreh $^{\mathrm{a}}, \mathrm{R} . \mathrm{Edziah}^{\mathrm{a}}$ \\ ${ }^{a}$ Department of Physics, College of Agriculture and Natural Sciences, University of Cape \\ Coast, PMB, Cape Coast, Ghana \\ ${ }^{b}$ Department of Mathematics, College of Agriculture and Natural Sciences, University of \\ Cape Coast, PMB, Cape Coast, Ghana \\ ${ }^{c}$ Department of Physics, The Pennsylvania State University Altoona, Altoona, \\ Pennsylvania 16601, U.S.A \\ ${ }^{d}$ Material Research Institute, The Pennsylvania State University, University Park, \\ Pennsylvania 16802, U.S.A
}

\begin{abstract}
Herein, we report on a giant thermoelectric figure of merit $(\mathcal{Z T})$ of a nondegenerate fluorine-doped single-walled carbon nanotube (FSWCNT) using a tractable analytical approach and the phonon lattice Boltzmann model (LBM). We investigate the influence of the doping concentration $\left(n_{o}\right)$, and the overlapping integrals $\left(\Delta_{s}\right.$ and $\left.\Delta_{z}\right)$ on the $\mathcal{Z T}$. The $\mathcal{Z} \mathcal{T}$ and the temperature range of operation can be tuned using the doping (impurity) concentration and the overlapping integrals, respectively. The lattice thermal conductivity obtained using the phonon LBM was calculated to be; $\kappa_{\ell}=107.2 \mathrm{~W} / \mathrm{mK}$ which yielded a $\mathcal{Z T}$ greater than 20. Interestingly, the $\mathcal{Z T}$ obtained is higher than what has been reported in superlattices, $(\mathcal{Z T} \approx 1.4)$ and superlattice nanowire, $(\mathcal{Z T} \approx 4)$ at $300 K$, making FSWCNT a potential candidate for thermoelectric applications.
\end{abstract} Keywords: carbon canotube, fluorine, figure-of-merit, thermal conductivity

*Corresponding author email: cxa269@psu.edu This work is licensed under the Creative Commons Attribution International License (CC BY). http://creativecommons.org/licenses/by/4.0/ 


\section{Introduction}

Conversion of energy between heat and electricity based on thermoelectric effects is indispensable in the energy mix in mitigating the global energy needs and climate change due to the potential applications in the area of waste heat recovery and refrigeration $[1,2]$. Practically, the phenomenon is used for electric power generation (Seebeck effect) or for cooling (Peltier effect) without mechanical moving parts [3-5]. The performance of a thermoelectric device is usually determined by its dimensionless figure-of-merit (FoM), $\mathcal{Z T}=\alpha^{2} \sigma T / \kappa$, where $\alpha, \sigma, T, \kappa$ are the Seebeck coefficient (thermoelectric power), the electrical conductivity, the absolute temperature, and the thermal conductivity (which comprises the electronic $\left(\kappa_{e}\right)$ and the lattice $\left(\kappa_{\ell}\right)$ components), respectively [39]. Enhancements in the $\mathcal{Z T}$ have been achieved in nanostructures, mainly due to the thermal conductivity reduction by phonon-boundary scattering as the amount of interfaces increases [10].

Theoretical studies and experimental investigations of the thermoelectric properties of low dimensional (1-D and 2-D) systems have been pursued extensively for various materials [8,11-13]. Quantum confinement of electrons are expected to lead to improved $\mathcal{Z} \mathcal{T}$ value in low-dimensional materials, due to the anticipated enhancement of FoM parameters. 0-D structures hold even greater promise than $1-\mathrm{D}$ and $2-\mathrm{D}$ systems [8]. However, unlike $1-\mathrm{D}$ and $2-\mathrm{D}$ systems, where at least one of the directions is not confined and thus, can provide electrical conduction, $0-\mathrm{D}$ structures such as quantum dots are confined in all directions and may pose difficulties for some applications [14-16]. For this reason, several novel structures based on quantum dots that capture the essence of $0-\mathrm{D}$ structures have been proposed and synthesized, such as quantum dot array superlattices [17] and superlattice nanowires [18]. These new structures have shown great promise. Harman et al. have measured an impressive thermoelectric performance with $\mathcal{Z} \mathcal{T} \approx 2$ at $300 K$ for $P b T e S e$ based quantum dot superlattices [17], compared to the highest $\mathcal{Z} \mathcal{T}$ of $\approx 1$ for conventional bulk materials. A possible mechanism for this enhancement is attributed to the mi- 
niband formation in coupled 3-D quantum dot arrays [19].

1-D nanostructures such as single-walled carbon nanotubes (SWCNTs) has attracted much attention due to their outstanding electrical conductivity. The dominant contribution to the thermoelectric power is purely electronic, though in some materials, such as graphite, an electron-phonon scattering contribution or phonon drag effect also becomes prominent at certain temperature ranges, especially at low temperature. However, even though the thermal properties of nanotubes are dominated by phonons, we expect that the thermoelectric power will be primarily governed by the electronic drift contribution, the electronic band-structure of the nanotubes, and the electron scattering mechanisms [7$9,20]$. SWCNTs skeletal structure enables the attachment of large number of foreign atoms to the surface without destroying the tubular structure. Doping an armchair-SWCNT with fluorine atoms changes its nature from metallic to semiconducting [8,21-25].

In this study, we calculate the Peltier coefficient, the zero-current electron density, the thermal conductivity and the FoM of the FSWCNT. We noted that the properties mentioned above strongly depend on the geometric chiral angle (GCA, $\theta_{h}$ ), temperature $(T)$ and real overlapping integrals for jumps along the tubular axis $\left(\Delta_{z}\right)$ and the base helix $\left(\Delta_{s}\right)$ [7-9]. The variation of these parameters can give rise to a giant thermoelectric power, unusual low electron and lattice thermal conductivities and a $\mathcal{Z} \mathcal{T}$ greater than 6 (and as high as 20), making FSWCNT a potential candidate for thermoelectric applications. To the best of our knowledge, no study has investigated the FoM in FSWCNTs. This study aims to investigate the thermoelectric FoM of FSWCNT with a non-parabolic double periodic band.

\section{Theory}

Carrier Current Densities

We consider the problem in the semi-classical regime using the Boltzmann transport equation (BTE) written as [6-8]:

$$
\frac{\partial f(\vec{r}, \vec{p}, t)}{\partial t}+v(\vec{p}) \cdot \nabla_{r} f(\vec{r}, \vec{p}, t)+e \vec{E} \nabla_{p} f(\vec{r}, \vec{p}, t)=-\frac{f(\vec{r}, \vec{p}, t)-f_{o}(\vec{p})}{\tau} .
$$


Here, $f(\vec{r}, \vec{p}, t)$ is the non-equilibrium carrier distribution function, $f_{o}(\vec{p})$ is the local equilibrium distribution function, $\vec{v}(\vec{p})$ is the carrier miniband velocity, $\vec{E}$ is the applied electric field, $\vec{r}$ is the carrier position, $\vec{p}$ is the quasi-momentum of the carrier, $\tau$ is the relaxation time and $e$ is the carrier charge. In the linear approximation, where the second term on the left hand side is considered to be a weak perturbation, the solution to the BTE is expressed as [6-9]:

$$
\begin{aligned}
f(p)= & \tau^{-1} \int_{0}^{\infty} \exp (-t / \tau) f_{o}\left(p-e \int_{0}^{\infty}\left[\vec{E}_{o}+\vec{E} \cos \left(\omega t^{\prime}\right)\right] d t^{\prime}\right) d t \\
+ & \int_{0}^{\infty} \exp (-t / \tau) d t\left\{\left[\varepsilon\left(p-e \int_{0}^{\infty}\left[\vec{E}_{o}+\vec{E} \cos \left(\omega t^{\prime}\right)-\mu\right] \frac{\nabla T}{T}+\nabla \mu\right\}\right.\right. \\
& \times v\left(p-e \int_{0}^{\infty}\left[\vec{E}_{o}+\vec{E} \cos \left(\omega t^{\prime}\right)\right) \frac{\partial f_{o}}{\partial \varepsilon}\left(p-e \int_{0}^{\infty}\left[\vec{E}_{o}+\vec{E} \cos \left(\omega t^{\prime}\right)\right]\right),\right.
\end{aligned}
$$

where $\mu$ is the electrochemical potential which ensures carrier conservation. For a $p$-type FSWCNT with a periodicity of $3 b$, the energy band is given as $[8,21$ $25]$ :

$$
\varepsilon(\vec{p})=\varepsilon_{o}-\Delta_{s} \cos \frac{u \vec{p}_{s} b_{s}}{\hbar}-\Delta_{z} \cos \frac{w \vec{p}_{z} b_{z}}{\hbar},
$$

where $u=\sqrt{3} / 2$ and $w=3 \sqrt{3} / 2 . \varepsilon_{o}$ is the energy of an outer-shell electron in an isolated carbon atom, $\Delta_{s}$ and $\Delta_{z}$ are the real overlapping integral for jumps along the respective coordinates, $\vec{p}_{s}$ and $\vec{p}_{z}$ are the carrier momentum along the base helix and the tubular axis, respectively. $b_{s}$ is the distance between the site $n$ and $n+1$ along the base helix and $b_{z}$ is the distance between the site $n$ and $n+N$ along the tubular axis. The carrier charge $(\vec{j})$ and thermal $(\vec{q})$ current densities are defined as [6-9]:

$$
\vec{j}=e \sum_{p} \vec{v}(\vec{p}) f(\vec{p}) \quad \vec{q}=\sum_{p}[\varepsilon(p)-\mu] \vec{v}(\vec{p}) f(\vec{p})
$$

The axial component of the carrier charge is obtained as [8]:

$$
\begin{aligned}
\vec{j}_{z}=\left(\sigma_{z}+\right. & \left.\sigma_{s} \sin ^{2} \theta_{h}\right) \nabla_{z}\left(\frac{\mu}{e}-\phi\right) \\
& -\sigma_{z} \frac{k_{B}}{e}\left[\left(\frac{\varepsilon_{o}-\mu}{k_{B} T}\right)-\Delta_{z}^{*} \frac{I_{o}\left(\Delta_{z}^{*}\right)}{I_{1}\left(\Delta_{z}\right)}+2-\Delta_{s}^{*} \frac{I_{1}\left(\Delta_{s}^{*}\right)}{I_{o}\left(\Delta_{s}^{*}\right)}\right] \nabla_{z} T \\
& \quad-\sigma_{s} \frac{k}{e}\left[\left(\frac{\varepsilon_{o}-\mu}{k_{B} T}\right)-\Delta_{s}^{*} \frac{I_{o}\left(\Delta_{s}^{*}\right)}{I_{1}\left(\Delta_{s}^{*}\right)}+2-\Delta_{z}^{*} \frac{I_{1}\left(\Delta_{z}\right)}{I_{o}\left(\Delta_{z}^{*}\right)}\right] \sin \theta_{h} \nabla_{s} T .
\end{aligned}
$$


and the circumferential component is expressed as [8]:

$$
\begin{aligned}
\vec{j}_{c} & =\sigma_{s} \sin \theta_{h} \cos \theta_{h} \nabla_{z}\left(\frac{\mu}{e}-\phi\right) \\
& -\sigma_{s} \frac{k_{B}}{e} \sin \theta_{h} \cos \theta_{h}\left[\left(\frac{\varepsilon_{o}-\mu}{k_{B} T}\right)-\Delta_{s}^{*} \frac{I_{o}\left(\Delta_{s}^{*}\right)}{I_{1}\left(\Delta_{s}^{*}\right)}+2-\Delta_{z}^{*} \frac{I_{1}\left(\Delta_{z}\right)}{I_{o}\left(\Delta_{z}^{*}\right)}\right] \nabla_{z} T .
\end{aligned}
$$

The carrier thermal current density is also obtained as:

$$
\begin{aligned}
& \vec{q}=\tau^{-1} \int_{0}^{-\infty} \exp \left(\frac{-t}{\tau}\right) d t \sum_{p}[\varepsilon(\vec{p})-\mu] \\
& \times \vec{v}(\vec{p}) f_{o}\left(\vec{p}-e \int_{t-t^{\prime}}^{t}\left[\vec{E}_{o}+\vec{E} \cos \omega t^{\prime}\right] d t^{\prime}\right)+\int_{0}^{-\infty} \exp \left(\frac{-t}{\tau}\right) d t \sum_{p}[\varepsilon(\vec{p})-\mu] \\
& \times \vec{v}(\vec{p})\left\{[\varepsilon(\vec{p})-\mu] \frac{\nabla T}{T}-\mu\right\} \times \vec{v}(\vec{p}) \cdot \frac{\partial f_{o}(\vec{p})}{\partial \varepsilon(\vec{p})}\left(\vec{p}-e \int_{t-t^{\prime}}^{t}\left[\vec{E}_{o}+\vec{E} \cos \omega t^{\prime}\right] d t^{\prime}\right) .
\end{aligned}
$$

We resolve Eq.(7) along the base helix $(\overrightarrow{\mathcal{S}})$ and the tubular axis $(\overrightarrow{\mathcal{Z}})$, by neglecting the interference between the axial and the helical paths connecting a pair of atoms, so that the transverse motion quantizatoin is ignored [7-9]. The carrier thermal components yields:

$$
\begin{aligned}
& \overrightarrow{\mathcal{Z}}_{q}=\tau^{-1} \int_{0}^{-\infty} \exp \left(\frac{-t}{\tau}\right) d t \sum_{p}\left[\varepsilon\left(\vec{p}-e \int_{t-t^{\prime}}^{t}\left[\vec{E}_{o}+\vec{E}_{z} \cos \omega t^{\prime}\right] d t^{\prime}\right)-\mu\right] \\
& \times \vec{v}_{z}\left(\vec{p}-e \int_{t-t^{\prime}}^{t}\left[\vec{E}_{o}+\vec{E}_{z} \cos \omega t^{\prime}\right] d t^{\prime}\right) f_{o}(\vec{p}) \\
& +\int_{0}^{-\infty} \exp \left(\frac{-t}{\tau}\right) d t \sum_{p}\left[\varepsilon\left(\vec{p}-e \int_{t-t^{\prime}}^{t}\left[\vec{E}_{o}+\vec{E}_{z} \cos \omega t^{\prime}\right] d t^{\prime}\right)-\mu\right] \\
& \times\left\{[\varepsilon(\vec{p})-\mu] \frac{\nabla_{z} T}{T}+\nabla_{z} \mu\right\} \vec{v}_{z}\left(\vec{p}-e \int_{t-t^{\prime}}^{t}\left[\vec{E}_{o}+\vec{E}_{z} \cos \omega t^{\prime}\right] d t^{\prime}\right) \cdot \frac{\partial f_{o}(\vec{p})}{\partial \varepsilon(p)} \vec{v}_{z}(\vec{p}),
\end{aligned}
$$

and

$$
\begin{aligned}
\overrightarrow{\mathcal{S}}_{q}=\tau^{-1} \int_{0}^{-\infty} & \exp \left(\frac{-t}{\tau}\right) d t \sum_{p}\left[\varepsilon\left(\vec{p}-e \int_{t-t^{\prime}}^{t}\left[\vec{E}_{o}+\vec{E}_{s} \cos \omega t^{\prime}\right] d t^{\prime}\right)-\mu\right] \\
& \times \vec{v}_{s}\left(\vec{p}-e \int_{t-t^{\prime}}^{t}\left[\vec{E}_{o}+\vec{E}_{s} \cos \omega t^{\prime}\right] d t^{\prime}\right) f_{o}(\vec{p}) \\
& +\int_{0}^{-\infty} \exp \left(\frac{-t}{\tau}\right) d t \sum_{p}\left[\varepsilon\left(\vec{p}-e \int_{t-t^{\prime}}^{t}\left[\vec{E}_{o}+\vec{E}_{s} \cos \omega t^{\prime}\right] d t^{\prime}\right)-\mu\right]
\end{aligned}
$$




$$
\times\left\{[\varepsilon(\vec{p})-\mu] \frac{\nabla_{s} T}{T}+\nabla_{s} \mu\right\} \vec{v}_{s}\left(\vec{p}-e \int_{t-t^{\prime}}^{t}\left[\vec{E}_{o}+\vec{E}_{s} \cos \omega t^{\prime}\right] d t^{\prime}\right) \cdot \frac{\partial f_{o}(\vec{p})}{\partial \varepsilon(\vec{p})} \vec{v}_{s}(\vec{p}) .
$$

For a non-degenerate electron gas, we use the Boltzmann equilibrium distribution function $f_{o}(p)$ expressed as [8]:

$$
f_{o}(p)=\frac{n_{o} u w b_{z} b_{s}}{2 I_{o}\left(\Delta_{s}^{*}\right) I_{o}\left(\Delta_{z}^{*}\right)} \exp \left[\Delta_{s}^{*} \cos \frac{u \vec{p}_{s} b_{s}}{\hbar}+\Delta_{z}^{*} \cos \frac{w \vec{p}_{z} b_{z}}{\hbar}\right],
$$

where $n_{o}$ is the electron concentration, $I_{n}(x)$ is the modified Bessel function of order $n$ and $k_{B}$ is Boltzmann's constant. The carrier miniband velocity components $\vec{v}_{s}$ and $\vec{v}_{z}$ are given in ref. [8], where the integration is carried out within the first Brillouin zone $-\pi \hbar / b_{z} \leq \vec{p}_{z} \leq \pi \hbar / b_{z}$ and $-\pi \hbar / b_{s} \leq \vec{p}_{s} \leq \pi \hbar / b_{s}$, respectively. Solving explicitly, we express the thermal current density into axial and circumferential components without a lost of generality as: $\vec{q}_{z}=$ $\overrightarrow{\mathcal{Z}}_{q}+\overrightarrow{\mathcal{S}}_{q} \sin \theta_{h}, \vec{q}_{s}=\overrightarrow{\mathcal{S}}_{q} \cos \theta_{h}$, respectively. The axial carrier thermal current density is given as:

$$
\begin{aligned}
& \vec{q}_{z}=\frac{k_{B} T}{e}\left\{\sigma _ { z } \left[\left(\frac{\varepsilon_{o}-\mu}{k_{B} T}\right) \sum_{n=-\infty} J_{n}^{2}(\chi)\right.\right. \\
& \left.-\frac{\Delta_{z}^{*}}{2}\left(1+3 \sum_{n=-\infty} J_{n}^{2}(\chi)\right)\left(\frac{I_{o}\left(\Delta_{z}^{*}\right)}{I_{1}\left(\Delta_{z}^{*}\right)}-\frac{2}{\Delta_{z}^{*}}\right)-\Delta_{s}^{*} \sum_{n=-\infty} J_{n}^{2}(\chi) \frac{I_{1}\left(\Delta_{s}^{*}\right)}{I_{o}\left(\Delta_{s}^{*}\right)}\right] \\
& +\sigma_{s} \sin ^{2} \theta_{h}\left[\left(\frac{\varepsilon_{o}-\mu}{k_{B} T}\right) \sum_{n=-\infty} J_{n}^{2}(\chi)-\frac{\Delta_{s}^{*}}{2}\left(1+3 \sum_{n=-\infty} J_{n}^{2}(\chi)\right)\left(\frac{I_{o}\left(\Delta_{s}^{*}\right)}{I_{1}\left(\Delta_{s}^{*}\right)}-\frac{2}{\Delta_{s}^{*}}\right)\right. \\
& -\frac{k_{B}^{2} T}{e^{2}}\left\{\sigma_{z}\left[\left(\frac{\varepsilon_{o}-\mu}{k_{B} T}\right)^{2} \sum_{n=-\infty} J_{n}^{2}(\chi) \frac{I_{1}\left(\Delta_{z}^{*}\right)}{I_{o}\left(\Delta_{z}^{*}\right)}\right]\right\} \nabla_{z}\left(\frac{\mu}{e}-\phi\right)-\frac{\Delta_{z}^{*}}{2}\left(\frac{\varepsilon_{o}-\mu}{k_{B} T}\right)\left(\frac{I_{o}\left(\Delta_{z}^{*}\right)}{I_{1}\left(\Delta_{z}^{*}\right)}-\frac{2}{\Delta_{z}^{*}}\right)\left(1+3 \sum_{n=-\infty} J_{n}^{2}(\chi)\right) \\
& -2 \Delta_{s}^{*}\left(\frac{\varepsilon_{o}-\mu}{k_{B} T}\right) \frac{I_{1}\left(\Delta_{s}^{*}\right)}{I_{o}\left(\Delta_{s}^{*}\right)} \sum_{n=-\infty} J_{n}^{2}(\chi)+\frac{\Delta_{z}^{* 2}}{2}\left(1-\frac{3 I_{o}\left(\Delta_{z}^{*}\right)}{\Delta_{z}^{*} I_{1}\left(\Delta_{z}^{*}\right)}+\frac{6}{\Delta_{z}^{* 2}}\right)\left(1+\sum_{n=-\infty} J_{n}^{2}(\chi)\right) \\
& \left.+\frac{\Delta_{s}^{*} \Delta_{z}^{*}}{2}\left(\frac{I_{o}\left(\Delta_{z}^{*}\right)}{I_{1}\left(\Delta_{z}^{*}\right)}-\frac{2}{\Delta_{z}^{*}}\right) \frac{I_{1}\left(\Delta_{s}^{*}\right)}{I_{o}\left(\Delta_{s}^{*}\right)}\left(1+3 \sum_{n=-\infty} J_{n}^{2}(\chi)\right)+\Delta_{s}^{* 2}\left(1-\frac{I_{1}\left(\Delta_{s}^{*}\right)}{\Delta_{s}^{*} I_{o}\left(\Delta_{s}^{*}\right)}\right) \sum_{n=-\infty} J_{n}^{2}(\chi)\right] \\
& +\sigma_{s} \sin ^{2} \theta_{h}\left[\left(\frac{\varepsilon_{o}-\mu}{k_{B} T}\right)^{2} \sum_{n=-\infty} J_{n}^{2}(\chi)-\frac{\Delta_{s}^{*}}{2}\left(\frac{\varepsilon_{o}-\mu}{k_{B} T}\right)\left(\frac{I_{o}\left(\Delta_{s}^{*}\right)}{I_{1}\left(\Delta_{s}^{*}\right)}-\frac{2}{\Delta_{s}^{*}}\right)\left(1+3 \sum_{n=-\infty} J_{n}^{2}(\chi)\right)\right.
\end{aligned}
$$




$$
\begin{aligned}
& -2 \Delta_{z}^{*}\left(\frac{\varepsilon_{o}-\mu}{k_{B} T}\right) \frac{I_{1}\left(\Delta_{z}^{*}\right)}{I_{o}\left(\Delta_{z}^{*}\right)} \sum_{n=-\infty} J_{n}^{2}(\chi)+\frac{\Delta_{s}^{* 2}}{2}\left(1-\frac{3 I_{o}\left(\Delta_{s}^{*}\right)}{\Delta_{s}^{*} I_{1}\left(\Delta_{s}^{*}\right)}+\frac{6}{\Delta_{s}^{* 2}}\right)\left(1+\sum_{n=-\infty} J_{n}^{2}(\chi)\right) \\
& \left.\left.+\frac{\Delta_{z}^{*} \Delta_{s}^{*}}{2}\left(\frac{I_{o}\left(\Delta_{s}^{*}\right)}{I_{1}\left(\Delta_{s}^{*}\right)}-\frac{2}{\Delta_{s}^{*}}\right) \frac{I_{1}\left(\Delta_{z}^{*}\right)}{I_{o}\left(\Delta_{z}^{*}\right)}\left(1+3 \sum_{n=-\infty} J_{n}^{2}(\chi)\right)+\Delta_{z}^{* 2}\left(1-\frac{I_{1}\left(\Delta_{z}^{*}\right)}{\Delta_{z}^{*} I_{o}\left(\Delta_{z}^{*}\right)}\right) \sum_{n=-\infty} J_{n}^{2}(\chi)\right]\right\} \nabla_{z} T,
\end{aligned}
$$

and circumferential thermal component yields:

$$
\begin{gathered}
\vec{q}_{c}=\sigma_{s} \frac{k_{B} T}{e}\left\{\left(\frac{\varepsilon_{o}-\mu}{k_{B} T}\right) \sum_{n=-\infty} J_{n}^{2}(\chi)-\frac{\Delta_{s}^{*}}{2}\left(1+3 \sum_{n=-\infty} J_{n}^{2}(\chi)\right)\left(\frac{I_{o}\left(\Delta_{s}^{*}\right)}{I_{1}\left(\Delta_{s}^{*}\right)}-\frac{2}{\Delta_{s}^{*}}\right)\right. \\
\left.-\Delta_{z}^{*} \sum_{n=-\infty} J_{n}^{2}(\chi) \frac{I_{1}\left(\Delta_{z}^{*}\right)}{I_{o}\left(\Delta_{z}^{*}\right)}\right\} \nabla_{z}\left(\frac{\mu}{e}-\phi\right) \sin \theta_{h} \cos \theta_{h} \\
-\sigma_{s} \frac{k_{B}^{2} T}{e^{2}}\left\{\left(\frac{\varepsilon_{o}-\mu}{k_{B} T}\right)^{2} \sum_{n=-\infty} J_{n}^{2}(\chi)-\frac{\Delta_{s}^{*}}{2}\left(\frac{\varepsilon_{o}-\mu}{k_{B} T}\right)\left(\frac{I_{o}\left(\Delta_{s}^{*}\right)}{I_{1}\left(\Delta_{s}^{*}\right)}-\frac{2}{\Delta_{s}^{*}}\right)\left(1+3 \sum_{n=-\infty} J_{n}^{2}(\chi)\right)\right. \\
-2 \Delta_{z}^{*}\left(\frac{\varepsilon_{o}-\mu}{k_{B} T}\right) \frac{I_{1}\left(\Delta_{z}^{*}\right)}{I_{o}\left(\Delta_{z}^{*}\right)} \sum_{n=-\infty} J_{n}^{2}(\chi)+\frac{\Delta_{s}^{* 2}}{2}\left(1-\frac{3 I_{o}\left(\Delta_{s}^{*}\right)}{\Delta_{s}^{*} I_{1}\left(\Delta_{s}^{*}\right)}+\frac{6}{\Delta_{s}^{* 2}}\right)\left(1+\sum_{n=-\infty} J_{n}^{2}(\chi)\right) \\
+\frac{\Delta_{z}^{*} \Delta_{s}^{*}}{2}\left(\frac{I_{o}\left(\Delta_{s}^{*}\right)}{I_{1}\left(\Delta_{s}^{*}\right)}-\frac{2}{\Delta_{s}^{*}}\right) \frac{I_{1}\left(\Delta_{z}^{*}\right)}{I_{o}\left(\Delta_{z}^{*}\right)}\left(1+3 \sum_{n=-\infty} J_{n}^{2}(\chi)\right) \\
\left.+\Delta_{z}^{* 2}\left(1-\frac{I_{1}\left(\Delta_{z}^{*}\right)}{\Delta_{z}^{*} I_{o}\left(\Delta_{z}^{*}\right)}\right) \sum_{n=-\infty} J_{n}^{2}(\chi)\right\} \nabla_{z} T \sin \theta_{h} \cos \theta_{h}, \quad(12)
\end{gathered}
$$

where $\vec{E}=\nabla_{z}(\mu / e-\phi)$. The representation of $\vec{q}$ in terms of $\vec{E}$ in Eq.(11) and Eq.(12) is not convinient for comparing theory with experiment; it is therefore worthy to express $\vec{q}$ in terms of $\vec{j}$ and $\nabla T$. Combining Eq.(11) and Eq.(12) and solving explicitly yields:

$$
\begin{gathered}
\vec{q}_{z}=\frac{k_{B}}{e}\left\{\frac { \sigma _ { z } } { ( \sigma _ { z } + \sigma _ { s } \operatorname { s i n } ^ { 2 } \theta _ { h } ) } \left[\left(\frac{\varepsilon_{o}-\mu}{k_{B} T}\right) \sum_{n=-\infty} J_{n}^{2}(\chi)\right.\right. \\
\left.-\frac{\Delta_{z}^{*}}{2}\left(1+3 \sum_{n=-\infty} J_{n}^{2}(\chi)\right)\left(\frac{I_{o}\left(\Delta_{z}^{*}\right)}{I_{1}\left(\Delta_{z}^{*}\right)}-\frac{2}{\Delta_{z}^{*}}\right)-\Delta_{s}^{*} \sum_{n=-\infty} J_{n}^{2}(\chi) \frac{I_{1}\left(\Delta_{s}^{*}\right)}{I_{o}\left(\Delta_{s}^{*}\right)}\right] \\
+\frac{\sigma_{s} \sin ^{2} \theta_{h}}{\left(\sigma_{z}+\sigma_{s} \sin ^{2} \theta_{h}\right)}\left[\left(\frac{\varepsilon_{o}-\mu}{k_{B} T}\right) \sum_{n=-\infty} J_{n}^{2}(\chi)-\frac{\Delta_{s}^{*}}{2}\left(1+3 \sum_{n=-\infty} J_{n}^{2}(\chi)\right)\left(\frac{I_{o}\left(\Delta_{s}^{*}\right)}{I_{1}\left(\Delta_{s}^{*}\right)}-\frac{2}{\Delta_{s}^{*}}\right)\right. \\
\left.\left.-\Delta_{z}^{*} \sum_{n=-\infty} J_{n}^{2}(\chi) \frac{I_{1}\left(\Delta_{z}^{*}\right)}{I_{o}\left(\Delta_{z}^{*}\right)}\right]\right\} T \vec{j}_{z}
\end{gathered}
$$




$$
\begin{aligned}
& -\frac{k_{B}^{2} T}{e^{2}}\left\{\sigma _ { z } \left[\left(\frac{\varepsilon_{o}-\mu}{k_{B} T}\right)^{2} \sum_{n=-\infty} J_{n}^{2}(\chi)-\frac{\Delta_{z}^{*}}{2}\left(\frac{\varepsilon_{o}-\mu}{k_{B} T}\right)\left(\frac{I_{o}\left(\Delta_{z}^{*}\right)}{I_{1}\left(\Delta_{z}^{*}\right)}-\frac{2}{\Delta_{z}^{*}}\right)\left(1+3 \sum_{n=-\infty} J_{n}^{2}(\chi)\right)\right.\right. \\
& -2 \Delta_{s}^{*}\left(\frac{\varepsilon_{o}-\mu}{k_{B} T}\right) \frac{I_{1}\left(\Delta_{s}^{*}\right)}{I_{o}\left(\Delta_{s}^{*}\right)} \sum_{n=-\infty} J_{n}^{2}(\chi)+\frac{\Delta_{z}^{* 2}}{2}\left(1-\frac{3 I_{o}\left(\Delta_{z}^{*}\right)}{\Delta_{z}^{*} I_{1}\left(\Delta_{z}^{*}\right)}+\frac{6}{\Delta_{z}^{* 2}}\right)\left(1+\sum_{n=-\infty} J_{n}^{2}(\chi)\right) \\
& \left.+\frac{\Delta_{s}^{*} \Delta_{z}^{*}}{2}\left(\frac{I_{o}\left(\Delta_{z}^{*}\right)}{I_{1}\left(\Delta_{z}^{*}\right)}-\frac{2}{\Delta_{z}^{*}}\right) \frac{I_{1}\left(\Delta_{s}^{*}\right)}{I_{o}\left(\Delta_{s}^{*}\right)}\left(1+3 \sum_{n=-\infty} J_{n}^{2}(\chi)\right)+\Delta_{s}^{* 2}\left(1-\frac{I_{1}\left(\Delta_{s}^{*}\right)}{\Delta_{s}^{*} I_{o}\left(\Delta_{s}^{*}\right)}\right) \sum_{n=-\infty} J_{n}^{2}(\chi)\right] \\
& +\sigma_{s} \sin ^{2} \theta_{h}\left[\left(\frac{\varepsilon_{o}-\mu}{k_{B} T}\right)^{2} \sum_{n=-\infty} J_{n}^{2}(\chi)-\frac{\Delta_{s}^{*}}{2}\left(\frac{\varepsilon_{o}-\mu}{k_{B} T}\right)\left(\frac{I_{o}\left(\Delta_{s}^{*}\right)}{I_{1}\left(\Delta_{s}^{*}\right)}-\frac{2}{\Delta_{s}^{*}}\right)\left(1+3 \sum_{n=-\infty} J_{n}^{2}(\chi)\right)\right. \\
& -2 \Delta_{z}^{*}\left(\frac{\varepsilon_{o}-\mu}{k_{B} T}\right) \frac{I_{1}\left(\Delta_{z}^{*}\right)}{I_{o}\left(\Delta_{z}^{*}\right)} \sum_{n=-\infty} J_{n}^{2}(\chi)+\frac{\Delta_{s}^{* 2}}{2}\left(1-\frac{3 I_{o}\left(\Delta_{s}^{*}\right)}{\Delta_{s}^{*} I_{1}\left(\Delta_{s}^{*}\right)}+\frac{6}{\Delta_{s}^{* 2}}\right)\left(1+\sum_{n=-\infty} J_{n}^{2}(\chi)\right) \\
& \left.+\frac{\Delta_{z}^{*} \Delta_{s}^{*}}{2}\left(\frac{I_{o}\left(\Delta_{s}^{*}\right)}{I_{1}\left(\Delta_{s}^{*}\right)}-\frac{2}{\Delta_{s}^{*}}\right) \frac{I_{1}\left(\Delta_{z}^{*}\right)}{I_{o}\left(\Delta_{z}^{*}\right)}\left(1+3 \sum_{n=-\infty} J_{n}^{2}(\chi)\right)+\Delta_{z}^{* 2}\left(1-\frac{I_{1}\left(\Delta_{z}^{*}\right)}{\Delta_{z}^{*} I_{o}\left(\Delta_{z}^{*}\right)}\right) \sum_{n=-\infty} J_{n}^{2}(\chi)\right] \\
& +\left(\sigma_{z}+\sigma_{s} \sin ^{2} \theta_{h}\right)\left\{\frac { \sigma _ { z } } { ( \sigma _ { z } + \sigma _ { s } \operatorname { s i n } ^ { 2 } \theta _ { h } ) } \left[\left(\frac{\varepsilon_{o}-\mu}{k_{B} T}\right) \sum_{n=-\infty} J_{n}^{2}(\chi)\right.\right. \\
& \left.\left.-\frac{\Delta_{z}^{*}}{2}\left(1+3 \sum_{n=-\infty} J_{n}^{2}(\chi)\right)\left(\frac{I_{o}\left(\Delta_{z}^{*}\right)}{I_{1}\left(\Delta_{z}^{*}\right)}-\frac{2}{\Delta_{z}^{*}}\right)-\Delta_{s}^{*} \sum_{n=-\infty} J_{n}^{2}(\chi) \frac{I_{1}\left(\Delta_{s}^{*}\right)}{I_{o}\left(\Delta_{s}^{*}\right)}\right]\right\} \\
& +\frac{\sigma_{s} \sin ^{2} \theta_{h}}{\left(\sigma_{z}+\sigma_{s} \sin ^{2} \theta_{h}\right)}\left[\left(\frac{\varepsilon_{o}-\mu}{k_{B} T}\right) \sum_{n=-\infty} J_{n}^{2}(\chi)\right. \\
& \left.-\frac{\Delta_{s}^{*}}{2}\left(1+3 \sum_{n=-\infty} J_{n}^{2}(\chi)\right)\left(\frac{I_{o}\left(\Delta_{s}^{*}\right)}{I_{1}\left(\Delta_{s}^{*}\right)}-\frac{2}{\Delta_{s}^{*}}\right)-\Delta_{z}^{*} \sum_{n=-\infty} J_{n}^{2}(\chi) \frac{I_{1}\left(\Delta_{z}^{*}\right)}{I_{o}\left(\Delta_{z}^{*}\right)}\right] \\
& \times\left\{\frac{\sigma_{z}}{\left(\sigma_{z}+\sigma_{s} \sin ^{2} \theta_{h}\right)}\left[\left(\frac{\varepsilon_{o}-\mu}{k_{B} T}\right)-\Delta_{z}^{*} \frac{I_{o}\left(\Delta_{z}^{*}\right)}{I_{1}\left(\Delta_{z}^{*}\right)}+2-\Delta_{s}^{*} \frac{I_{1}\left(\Delta_{s}^{*}\right)}{I_{o}\left(\Delta_{s}^{*}\right)}\right]\right. \\
& \left.\left.+\frac{\sigma_{s} \sin ^{2} \theta_{h}}{\left(\sigma_{z}+\sigma_{s} \sin ^{2} \theta_{h}\right)}\left[\left(\frac{\varepsilon_{o}-\mu}{k_{B} T}\right)-\Delta_{s}^{*} \frac{I_{o}\left(\Delta_{s}^{*}\right)}{I_{1}\left(\Delta_{s}^{*}\right)}+2-\Delta_{z}^{*} \frac{I_{1}\left(\Delta_{z}^{*}\right)}{I_{o}\left(\Delta_{z}^{*}\right)}\right]\right\}\right\} \nabla_{z} T,
\end{aligned}
$$

and

$$
\begin{aligned}
& \vec{q}_{c}=\frac{k_{B}}{e}\left\{\left(\frac{\varepsilon_{o}-\mu}{k_{B} T}\right) \sum_{n=-\infty} J_{n}^{2}(\chi)\right. \\
& \left.-\frac{\Delta_{s}^{*}}{2}\left(1+3 \sum_{n=-\infty} J_{n}^{2}(\chi)\right)\left(\frac{I_{o}\left(\Delta_{s}^{*}\right)}{I_{1}\left(\Delta_{s}^{*}\right)}-\frac{2}{\Delta_{s}^{*}}\right)-\Delta_{z}^{*} \sum_{n=-\infty} J_{n}^{2}(\chi) \frac{I_{1}\left(\Delta_{z}^{*}\right)}{I_{o}\left(\Delta_{z}^{*}\right)}\right\} T \vec{j}_{c} \\
& -\sigma_{s} \frac{k_{B}^{2} T}{e^{2}} \sin \theta_{h} \cos \theta_{h}\left\{\left\{\left(\frac{\varepsilon_{o}-\mu}{k_{B} T}\right)^{2} \sum_{n=-\infty} J_{n}^{2}(\chi)-\frac{\Delta_{s}^{*}}{2}\left(\frac{\varepsilon_{o}-\mu}{k_{B} T}\right)\left(\frac{I_{o}\left(\Delta_{s}^{*}\right)}{I_{1}\left(\Delta_{s}^{*}\right)}-\frac{2}{\Delta_{s}^{*}}\right)\left(1+3 \sum_{n=-\infty} J_{n}^{2}(\chi)\right)\right.\right.
\end{aligned}
$$




$$
\begin{gathered}
-2 \Delta_{z}^{*}\left(\frac{\varepsilon_{o}-\mu}{k_{B} T}\right) \frac{I_{1}\left(\Delta_{z}^{*}\right)}{I_{o}\left(\Delta_{z}^{*}\right)} \sum_{n=-\infty} J_{n}^{2}(\chi)+\frac{\Delta_{s}^{* 2}}{2}\left(1-\frac{3 I_{o}\left(\Delta_{s}^{*}\right)}{\Delta_{s}^{*} I_{1}\left(\Delta_{s}^{*}\right)}+\frac{6}{\Delta_{s}^{* 2}}\right)\left(1+\sum_{n=-\infty} J_{n}^{2}(\chi)\right) \\
\left.+\frac{\Delta_{z}^{*} \Delta_{s}^{*}}{2}\left(\frac{I_{o}\left(\Delta_{s}^{*}\right)}{I_{1}\left(\Delta_{s}^{*}\right)}-\frac{2}{\Delta_{s}^{*}}\right) \frac{I_{1}\left(\Delta_{z}^{*}\right)}{I_{o}\left(\Delta_{z}^{*}\right)}\left(1+3 \sum_{n=-\infty} J_{n}^{2}(\chi)\right)+\Delta_{z}^{* 2}\left(1-\frac{I_{1}\left(\Delta_{z}^{*}\right)}{\Delta_{z}^{*} I_{o}\left(\Delta_{z}^{*}\right)}\right) \sum_{n=-\infty} J_{n}^{2}(\chi)\right\} \\
-\left\{\left(\frac{\varepsilon_{o}-\mu}{k_{B} T}\right) \sum_{n=-\infty} J_{n}^{2}(\chi)-\frac{\Delta_{s}^{*}}{2}\left(\frac{I_{o}\left(\Delta_{s}^{*}\right)}{I_{1}\left(\Delta_{s}^{*}\right)}-\frac{2}{\Delta_{s}^{*}}\right)\left(1+3 \sum_{n=-\infty} J_{n}^{2}(\chi)\right)-\Delta_{z}^{*} \sum_{n=-\infty} J_{n}^{2}(\chi) \frac{I_{1}\left(\Delta_{z}^{*}\right)}{I_{o}\left(\Delta_{z}^{*}\right)}\right\} \\
\left.\times\left[\left(\frac{\varepsilon_{o}-\mu}{k_{B} T}\right)-\Delta_{s}^{*} \frac{I_{o}\left(\Delta_{s}^{*}\right)}{I_{1}\left(\Delta_{s}^{*}\right)}+2-\Delta_{z}^{*} \frac{I_{1}\left(\Delta_{z}^{*}\right)}{I_{o}\left(\Delta_{z}^{*}\right)}\right]\right\} \nabla_{z} T . \quad(14)
\end{gathered}
$$

Eq.(13) and Eq.(14) are in the form of the Onsager relation as:

$$
\vec{q}_{c}=\Pi_{c z} \vec{j}_{c}-\kappa_{c z} \alpha_{c z} \nabla_{z} T \quad \vec{q}_{z}=\Pi_{z z} \vec{j}_{z}-\kappa_{z z} \alpha_{z z} \nabla_{z} T
$$

where $\kappa$ is the carrier thermal conductivity when the carrier current density is zero $(\vec{j}=0)$ and $\Pi$ is the Peltier coefficient $(\Pi=\alpha T)$. Comparing Eq.(13) and Eq.(14) to Eq.(15), the axial $\left(\Pi_{z z}\right)$ and circumferential $\left(\Pi_{c z}\right)$ components of the Peltier coefficients are respectively, given as:

$$
\begin{gathered}
\Pi_{z z}=\frac{k_{B}}{e}\left\{\frac { \sigma _ { z } } { ( \sigma _ { z } + \sigma _ { s } \operatorname { s i n } ^ { 2 } \theta _ { h } ) } \left[\left(\frac{\varepsilon_{o}-\mu}{k_{B} T}\right) \sum_{n=-\infty} J_{n}^{2}(\chi)\right.\right. \\
\left.-\frac{\Delta_{z}^{*}}{2}\left(1+3 \sum_{n=-\infty} J_{n}^{2}(\chi)\right)\left(\frac{I_{o}\left(\Delta_{z}^{*}\right)}{I_{1}\left(\Delta_{z}^{*}\right)}-\frac{2}{\Delta_{z}^{*}}\right)-\Delta_{s}^{*} \sum_{n=-\infty} J_{n}^{2}(\chi) \frac{I_{1}\left(\Delta_{s}^{*}\right)}{I_{o}\left(\Delta_{s}^{*}\right)}\right] \\
+\frac{\sigma_{s} \sin ^{2} \theta_{h}}{\left(\sigma_{z}+\sigma_{s} \sin ^{2} \theta_{h}\right)}\left[\left(\frac{\varepsilon_{o}-\mu}{k_{B} T}\right) \sum_{n=-\infty} J_{n}^{2}(\chi)\right. \\
\left.\left.-\frac{\Delta_{s}^{*}}{2}\left(1+3 \sum_{n=-\infty} J_{n}^{2}(\chi)\right)\left(\frac{I_{o}\left(\Delta_{s}^{*}\right)}{I_{1}\left(\Delta_{s}^{*}\right)}-\frac{2}{\Delta_{s}^{*}}\right)-\Delta_{z}^{*} \sum_{n=-\infty} J_{n}^{2}(\chi) \frac{I_{1}\left(\Delta_{z}^{*}\right)}{I_{o}\left(\Delta_{z}^{*}\right)}\right]\right\} T
\end{gathered}
$$

and

$$
\begin{aligned}
\Pi_{c z}=\frac{k_{B}}{e}\left\{\left(\frac{\varepsilon_{o}-\mu}{k_{B} T}\right) \sum_{n=-\infty} J_{n}^{2}(\chi)-\frac{\Delta_{s}^{*}}{2}\right. & \left(1+3 \sum_{n=-\infty} J_{n}^{2}(\chi)\right)\left(\frac{I_{o}\left(\Delta_{s}^{*}\right)}{I_{1}\left(\Delta_{s}^{*}\right)}-\frac{2}{\Delta_{s}^{*}}\right) \\
& \left.-\Delta_{z}^{*} \sum_{n=-\infty} J_{n}^{2}(\chi) \frac{I_{1}\left(\Delta_{z}^{*}\right)}{I_{o}\left(\Delta_{z}^{*}\right)}\right\} T .
\end{aligned}
$$

The expression and evaluation of the axial and circumferential component of the electrical conductivity $(\sigma)$, and the thermoelectric power $(\alpha)$ of the FSWCNT 
were reported in ref.[8]. The axial $\left(\kappa_{z z}\right)$ and the circumferential component $\left(\kappa_{c z}\right)$ of the carrier thermal conductivities are expressed as:

$$
\begin{aligned}
& \kappa_{z z}=\frac{k_{B}^{2} T}{e^{2}}\left\{\sigma _ { z } \left[\left(\frac{\varepsilon_{o}-\mu}{k_{B} T}\right)^{2} \sum_{n=-\infty} J_{n}^{2}(\chi)\right.\right. \\
& -\frac{\Delta_{z}^{*}}{2}\left(\frac{\varepsilon_{o}-\mu}{k_{B} T}\right)\left(\frac{I_{o}\left(\Delta_{z}^{*}\right)}{I_{1}\left(\Delta_{z}^{*}\right)}-\frac{2}{\Delta_{z}^{*}}\right)\left(1+3 \sum_{n=-\infty} J_{n}^{2}(\chi)\right)-2 \Delta_{s}^{*}\left(\frac{\varepsilon_{o}-\mu}{k_{B} T}\right) \frac{I_{1}\left(\Delta_{s}^{*}\right)}{I_{o}\left(\Delta_{s}^{*}\right)} \sum_{n=-\infty} J_{n}^{2}(\chi) \\
& +\frac{\Delta_{z}^{* 2}}{2}\left(1-\frac{3 I_{o}\left(\Delta_{z}^{*}\right)}{\Delta_{z}^{*} I_{1}\left(\Delta_{z}^{*}\right)}+\frac{6}{\Delta_{z}^{* 2}}\right)\left(1+\sum_{n=-\infty} J_{n}^{2}(\chi)\right) \\
& \left.+\frac{\Delta_{s}^{*} \Delta_{z}^{*}}{2}\left(\frac{I_{o}\left(\Delta_{z}^{*}\right)}{I_{1}\left(\Delta_{z}^{*}\right)}-\frac{2}{\Delta_{z}^{*}}\right) \frac{I_{1}\left(\Delta_{s}^{*}\right)}{I_{o}\left(\Delta_{s}^{*}\right)}\left(1+3 \sum_{n=-\infty} J_{n}^{2}(\chi)\right)+\Delta_{s}^{* 2}\left(1-\frac{I_{1}\left(\Delta_{s}^{*}\right)}{\Delta_{s}^{*} I_{o}\left(\Delta_{s}^{*}\right)}\right) \sum_{n=-\infty} J_{n}^{2}(\chi)\right] \\
& +\sigma_{s} \sin ^{2} \theta_{h}\left[\left(\frac{\varepsilon_{o}-\mu}{k_{B} T}\right)^{2} \sum_{n=-\infty} J_{n}^{2}(\chi)-\frac{\Delta_{s}^{*}}{2}\left(\frac{\varepsilon_{o}-\mu}{k_{B} T}\right)\left(\frac{I_{o}\left(\Delta_{s}^{*}\right)}{I_{1}\left(\Delta_{s}^{*}\right)}-\frac{2}{\Delta_{s}^{*}}\right)\left(1+3 \sum_{n=-\infty} J_{n}^{2}(\chi)\right)\right. \\
& -2 \Delta_{z}^{*}\left(\frac{\varepsilon_{o}-\mu}{k_{B} T}\right) \frac{I_{1}\left(\Delta_{z}^{*}\right)}{I_{o}\left(\Delta_{z}^{*}\right)} \sum_{n=-\infty} J_{n}^{2}(\chi)+\frac{\Delta_{s}^{* 2}}{2}\left(1-\frac{3 I_{o}\left(\Delta_{s}^{*}\right)}{\Delta_{s}^{*} I_{1}\left(\Delta_{s}^{*}\right)}+\frac{6}{\Delta_{s}^{* 2}}\right)\left(1+\sum_{n=-\infty} J_{n}^{2}(\chi)\right) \\
& \left.+\frac{\Delta_{z}^{*} \Delta_{s}^{*}}{2}\left(\frac{I_{o}\left(\Delta_{s}^{*}\right)}{I_{1}\left(\Delta_{s}^{*}\right)}-\frac{2}{\Delta_{s}^{*}}\right) \frac{I_{1}\left(\Delta_{z}^{*}\right)}{I_{o}\left(\Delta_{z}^{*}\right)}\left(1+3 \sum_{n=-\infty} J_{n}^{2}(\chi)\right)+\Delta_{z}^{* 2}\left(1-\frac{I_{1}\left(\Delta_{z}^{*}\right)}{\Delta_{z}^{*} I_{o}\left(\Delta_{z}^{*}\right)}\right) \sum_{n=-\infty} J_{n}^{2}(\chi)\right] \\
& +\left(\sigma_{z}+\sigma_{s} \sin ^{2} \theta_{h}\right)\left\{\frac { \sigma _ { z } } { \sigma _ { z } + \sigma _ { s } \operatorname { s i n } ^ { 2 } \theta _ { h } } \left[\left(\frac{\varepsilon_{o}-\mu}{k_{B} T}\right) \sum_{n=-\infty} J_{n}^{2}(\chi)\right.\right. \\
& \left.-\frac{\Delta_{z}^{*}}{2}\left(1+3 \sum_{n=-\infty} J_{n}^{2}(\chi)\right)\left(\frac{I_{o}\left(\Delta_{z}^{*}\right)}{I_{1}\left(\Delta_{z}^{*}\right)}-\frac{2}{\Delta_{z}^{*}}\right)-\Delta_{s}^{*} \sum_{n=-\infty} J_{n}^{2}(\chi) \frac{I_{1}\left(\Delta_{s}^{*}\right)}{I_{o}\left(\Delta_{s}^{*}\right)}\right] \\
& +\frac{\sigma_{s} \sin ^{2} \theta_{h}}{\sigma_{z}+\sigma_{s} \sin ^{2} \theta_{h}}\left[\left(\frac{\varepsilon_{o}-\mu}{k_{B} T}\right) \sum_{n=-\infty} J_{n}^{2}(\chi)\right] \\
& \left.\left.-\frac{\Delta_{s}^{*}}{2}\left(1+3 \sum_{n=-\infty} J_{n}^{2}(\chi)\right)\left(\frac{I_{o}\left(\Delta_{s}^{*}\right)}{I_{1}\left(\Delta_{s}^{*}\right)}-\frac{2}{\Delta_{s}^{*}}\right)-\Delta_{z}^{*} \sum_{n=-\infty} J_{n}^{2}(\chi) \frac{I_{1}\left(\Delta_{z}^{*}\right)}{I_{o}\left(\Delta_{z}^{*}\right)}\right\}\right\}
\end{aligned}
$$

and

$$
\begin{aligned}
& \kappa_{c z}=\sigma_{s} \frac{k_{B}^{2} T}{e^{2}} \sin \theta_{h} \cos \theta_{h}\left[\left\{\left(\frac{\varepsilon_{o}-\mu}{k_{B} T}\right)^{2} \sum_{n=-\infty} J_{n}^{2}(\chi)\right.\right. \\
& -\frac{\Delta_{s}^{*}}{2}\left(\frac{\varepsilon_{o}-\mu}{k_{B} T}\right)\left(\frac{I_{o}\left(\Delta_{s}^{*}\right)}{I_{1}\left(\Delta_{s}^{*}\right)}-\frac{2}{\Delta_{s}^{*}}\right)\left(1+3 \sum_{n=-\infty} J_{n}^{2}(\chi)\right)-2 \Delta_{z}^{*}\left(\frac{\varepsilon_{o}-\mu}{k_{B} T}\right) \frac{I_{1}\left(\Delta_{z}^{*}\right)}{I_{o}\left(\Delta_{z}^{*}\right)} \sum_{n=-\infty} J_{n}^{2}(\chi)
\end{aligned}
$$




$$
\begin{gathered}
+\frac{\Delta_{s}^{* 2}}{2}\left(1-\frac{3 I_{o}\left(\Delta_{s}^{*}\right)}{\Delta_{s}^{*} I_{1}\left(\Delta_{s}^{*}\right)}+\frac{6}{\Delta_{s}^{* 2}}\right)\left(1+\sum_{n=-\infty} J_{n}^{2}(\chi)\right) \\
\left.+\frac{\Delta_{z}^{*} \Delta_{s}^{*}}{2}\left(\frac{I_{o}\left(\Delta_{s}^{*}\right)}{I_{1}\left(\Delta_{s}^{*}\right)}-\frac{2}{\Delta_{s}^{*}}\right) \frac{I_{1}\left(\Delta_{z}^{*}\right)}{I_{o}\left(\Delta_{z}^{*}\right)}\left(1+3 \sum_{n=-\infty} J_{n}^{2}(\chi)\right)+\Delta_{z}^{* 2}\left(1-\frac{I_{1}\left(\Delta_{z}^{*}\right)}{\Delta_{z}^{*} I_{o}\left(\Delta_{z}^{*}\right)}\right) \sum_{n=-\infty} J_{n}^{2}(\chi)\right\} \\
\left.-\left\{\left(\frac{\varepsilon_{o}-\mu}{k_{B} T}\right) \sum_{n=-\infty} J_{n}^{2}(\chi)-\frac{\Delta_{s}^{*}}{2}\left(\frac{I_{o}\left(\Delta_{s}^{*}\right)}{I_{1}\left(\Delta_{s}^{*}\right)}-\frac{2}{\Delta_{s}^{*}}\right)\left(1+3 \sum_{n=-\infty} J_{n}^{2}(\chi)\right)-\Delta_{z}^{*} \sum_{n=-\infty} J_{n}^{2}(\chi) \frac{I_{1}\left(\Delta_{z}^{*}\right)}{I_{o}\left(\Delta_{z}^{*}\right)}\right\}\right] .
\end{gathered}
$$

\section{Lattice Thermal Conductivity $\left(\kappa_{\ell}\right)$}

Following the approach in ref.[26], we establish the phonon lattice Boltzmann model (LBM) which is obtained from the BTE for the kinetic theory of phonons. The solution to the LBM is conducted using Chapman-Enskog expansion. In the diffusive limit, the macroscopic heat transport equation is obtained and the correlation between the numerical parameters and the bulk material properties are established for 1D, 2D and 3D situations, respectively. In the hydrodynamic regime, $q \ell \ll 1$, the phonon LBM is obtained in discrete form [26,27-30] as:

$$
\frac{\partial \epsilon_{i}(\vec{x}, t)}{\partial t}+\vec{c}_{i} \cdot \nabla_{r} \epsilon_{i}(\vec{x}, t)=-\frac{\epsilon_{i}(\vec{x}, t)-\epsilon_{i}^{e q}(\vec{x}, t)}{\tau_{R}},
$$

where $\vec{c}_{i}(i=1,2 \ldots . n)$ are the discrete velocities. The spatial and time derivatives of $\epsilon_{i}(\vec{x}, t)$ on the left side of Eq.(20) are approximated to a dimensionless first order finite difference by replacing $\delta \vec{x}=\vec{c}_{i} \delta t$, where $\delta \vec{x}$ is the spatial step, $\delta t$ is the time step and $\tau=\tau_{R} / \delta t$ which yields:

$$
\frac{\epsilon_{i}\left(\vec{x}+\vec{c}_{i} \delta t, t+\delta t\right)-\epsilon_{i}(\vec{x}, t)}{\delta t}=-\frac{\epsilon_{i}(\vec{x}, t)-\epsilon_{i}^{e q}(\vec{x}, t)}{\tau},
$$

The discrete equilibrium phonon energy densities are obtained as:

$$
\epsilon_{i}^{e q}=\frac{1}{n} \int \hbar \omega \frac{D(\omega) d \omega}{\exp \left(\hbar \omega / k_{B} T\right)-1} .
$$

with $D(\omega)$ as the phonon density of states which incooperates transverse, longitudinal and in-plane branches. Employing Debye's approximation and after much simplication, the equilibrium phonon energy density reduces to:

$$
\epsilon^{e q}=\frac{3 \mathcal{N} k_{B} T}{V}\left(\frac{T}{\Theta_{D}}\right)^{3} \int_{0}^{\Theta_{D} / T} \frac{x^{3} d x}{\exp (x)-1} .
$$


Here, $\mathcal{N} / V$ is the number density of crystal atoms, $\Theta_{D}$ is the Debye temperature and $x \equiv \hbar \omega / k_{B} T$. The local temperature of the FSWCNT must be determined by an inverse numerical integration from the local phonon energy density based on Eq.(23). This inverse computation is complicated and only necessary for very low temperature condition $\left(T \ll \Theta_{D}\right)$, where the temperature dependence on the heat capacity is dominant for mathematical simplicity and tractable interpretation of results. In this study, high temperature limits are considered as well as in situations where the heat capacity approaches a constant, such that Eq.(23) reduces to $\epsilon^{e q} \approx c_{v} T$ (where $c_{v}$ is the heat capacity per unit volume). Thus, Eq.(22) simplifies as: $e_{i}^{e q}=e^{e q} / n=c_{v} T / n$, where

$$
c_{v}=\frac{3 \mathcal{N} k_{B}}{V}\left(\frac{T}{\Theta_{D}}\right)^{3} \int_{0}^{\Theta_{D} / T} \frac{x^{3} d x}{\exp (x)-1} .
$$

The phonon contribution is obtained by integrating over the phonon density of states with a convolution factor that reflects the energy and occupation of each phonon state. For nonzero temperatures, the convolution factor is 1 at $\omega=0$, and decreases smoothly to a value $\approx 0.1$ at $\hbar \omega=k_{B} T / 6$, so that the specific heat rises with temperature as the phonon states are occupied. This is because $D(\omega)$ is a complicated function of $\omega$ and thus, the specific heat at moderate temperatures, cannot be calculated analytically. At low temperature $\left(T \ll \Theta_{D}\right)$, however, the temperature dependence of the specific heat is in general much simpler. In this regime, the upper bound in Eq.(24) can be taken as infinity, and $D(\omega)$ is dominated by acoustic phonon modes, i.e. those with $\omega \rightarrow 0$ as $q \rightarrow 0$. If we consider a single acoustic mode in $d$ dimensions that obeys a dispersion relation $\omega \propto q^{\alpha}$, then from Eq.(24) it follows that: $c_{v} \propto T^{d / \alpha}$ for $T \ll \Theta_{D}$. Thus, the low-temperature specific heat contains information about both the dimensionality of the system and the phonon dispersion [31-34].

The different types of lattice structures for the phonon LBM simulation are shown in Fig. 1 with their probability distribution functions and their velocities. 


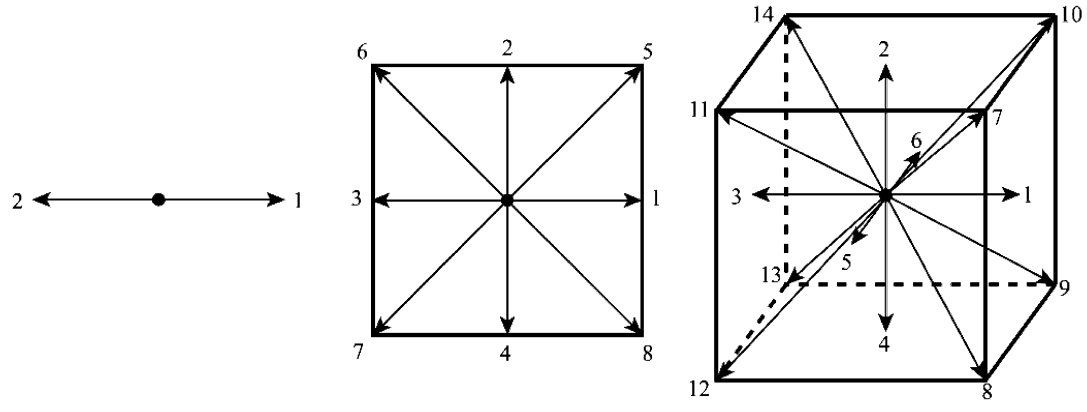

Figure 1: Lattice structures for phonon lattice Boltzmann model at different dimensions: (a) D1Q2, (b) D2Q8, (c) D3Q14 [26]

$$
c_{i}= \begin{cases}c\left(\cos \left(\frac{(i-1) \pi}{2}\right), \sin \left(\frac{(i-1) \pi}{2}\right)\right), & i=1,2,3,4 \\ \sqrt{2} c\left(\cos \left(\frac{(i-5) \pi}{2}+\frac{\pi}{4}\right), \sin \left(\frac{(i-5) \pi}{2}+\frac{\pi}{4}\right)\right), & i=5,6,7,8 .\end{cases}
$$

The discrete velocities are used to determine appropriate weight factors, $w_{i}$, through tensor moment expansions [27]. Along with maintaining consistent phonon propagation in each direction, weight factors are needed to preserve rotational invariance, meaning the lattice can be rotated in $90^{\circ}$ increments without changing the results. This is a necessary feature in LBM because the streaming operation is the same in each direction. A rotationally invariant cartesian lattice must satisfy the conservation equations, which are moments about the lattice speed [31-34].

In order to recover the macroscopic heat transport equation, we apply a Chapman-Enskog expansion to solve the phonon lattice Boltzmann equation in Eq.(21). The central-point component with vanishing lattice velocity is not accounted for in this formalism. This distinction originates from the fact that the conservation laws of particle number and mass in hydrodynamics are no longer ensured in phonon transport $[35,36]$ since phonons can be easily created and destroyed. However, phonons are bosons with zero static mass and so their energy as well as velocity can never reach zero; otherwise they will disappear, which is a basic precept of the classical harmonic oscillator: $\epsilon=\hbar \omega / 2(n+1 / 2)$. For a typical D2Q8 lattice, the Chapman-Enskog expansion can be done through similar procedures for other lattice structures. Expanding $\epsilon_{i}\left(\vec{x}+\vec{c}_{i} \delta t, t+\delta t\right)$ 
around $\epsilon_{i}(\vec{x}, t)$ using Taylor series within second order for small lattice time step, we obtain:

$$
\begin{aligned}
\epsilon_{i}\left(\vec{x}+\vec{c}_{i} \delta t, t+\delta t\right)=\epsilon_{i}(\vec{x}, t)+\delta t\left(\frac{\partial}{\partial t}+\vec{c}_{i \alpha} \frac{\partial}{\partial x_{\alpha}}\right) \epsilon_{i}(\vec{x}, t) \\
+\frac{1}{2}(\delta t)^{2}\left(\frac{\partial}{\partial t}+\vec{c}_{i \alpha} \frac{\partial}{\partial x_{\alpha}}\right)^{2} \epsilon_{i}(\vec{x}, t) .
\end{aligned}
$$

Substituting Eq.(26) into Eq.(21) yields:

$$
\left(\frac{\partial}{\partial t}+\vec{c}_{i \alpha} \frac{\partial}{\partial x_{\alpha}}\right) \epsilon_{i}(\vec{x}, t)+\frac{1}{2}(\delta t)\left(\frac{\partial}{\partial t}+\vec{c}_{i \alpha} \frac{\partial}{\partial x_{\alpha}}\right)^{2} \epsilon_{i}(\vec{x}, t)=-\frac{\epsilon_{i}(\vec{x}, t)-\epsilon_{i}^{e q}(\vec{x}, t)}{\tau_{R}}
$$

Expandng $\partial / \partial t$ and introducing two time scales $t_{1}$ and $t_{2}$ and one spatial scale $\vec{x}_{1}$, yields:

$$
\frac{\partial}{\partial x_{\alpha}}=\varepsilon \frac{\partial}{\partial x_{1 \alpha}} \quad \frac{\partial}{\partial t}=\varepsilon \frac{\partial}{\partial t_{1}}+\varepsilon^{2} \frac{\partial}{\partial t_{2}}
$$

where $\varepsilon$ is a small factor often chosen as the Knudson number, $K n$, (ratio of the phonon mean free path, $\ell$, to the characteristic length of the FSWCNT size $L$ ). The discrete phonon energy densities are asymptotically expanded within first order as: $\epsilon_{i}(\vec{x}, t)=\epsilon_{i}^{(0)}(\vec{x}, t)+\varepsilon \epsilon_{i}^{(1)}(\vec{x}, t)+\ldots$. Substituting this expression into Eq.(28) yields the subsequent orders of magnitude i.e. $\varepsilon^{0}, \varepsilon^{1}, \varepsilon^{2}$, respectively as:

$$
\begin{gathered}
\epsilon_{i}^{(0)}(\vec{x}, t)=\epsilon_{i}^{e q}(\vec{x}, t), \\
\frac{\partial \epsilon_{i}^{(0)}(\vec{x}, t)}{\partial t_{1}}+c_{i \alpha} \frac{\partial \epsilon_{i}^{(0)}(\vec{x}, t)}{\partial x_{1 \alpha}}=-\frac{1}{\tau_{R}} \epsilon_{i}^{(1)}(\vec{x}, t), \\
\frac{\partial \epsilon_{i}^{(0)}(\vec{x}, t)}{\partial t_{2}}+\left(1-\frac{1}{2 \tau}\right) \frac{\partial \epsilon_{i}^{(1)}(\vec{x}, t)}{\partial t_{1}}+\left(1-\frac{1}{2 \tau}\right) c_{i \alpha} \frac{\partial \epsilon_{i}^{(1)}(\vec{x}, t)}{\partial x_{1 \alpha}}=0 .
\end{gathered}
$$

The heat flux $\vec{q}$, is asymptotically expanded within first order as:

$$
\vec{q}_{\alpha}(\vec{x}, t)=\vec{q}_{\alpha}^{(0)}(\vec{x}, t)+\varepsilon \vec{q}_{\alpha}^{(1)}(\vec{x}, t)+\ldots \ldots
$$

Summing over $i$ of Eq.(29) and Eq.(31), respectively and combining the two equations in terms of space and time $t_{1}, t_{2}$ and $\vec{x}_{1}$ yields the energy balance equation which is expressed as:

$$
\frac{\partial \epsilon}{\partial t^{\prime}}+\frac{\partial q_{\alpha}}{\partial x_{\alpha}}=0
$$


with a realistic time scale $t^{\prime}$, related to the time scale $t$, in lattice Boltzmann model by $t^{\prime}=(1-1 / 2 \tau) t$. The macroscopic parameters i.e. local temperature and heat flux are computed from the discrete phonon energy density through:

$$
\epsilon=\sum_{i} \epsilon_{i}=c_{v} T \quad q_{\alpha}(\vec{x}, t)=\sum_{i} c_{i \alpha} \epsilon_{i}(\vec{x}, t)
$$

Multiplying both sides of Eq.(30) and Eq.(31) by $c_{i}$, and summing over $i$, and combining the two equations gives rise to the heat flux:

$$
q_{\alpha}=-\frac{3}{4} \tau_{R} c^{2} \frac{\partial \epsilon}{\partial x_{\alpha}}
$$

where $d \epsilon=c_{v} d T$ and the lattice thermal conductivity is given as:

$$
\kappa_{\ell}=c_{v} \tau_{R} \frac{v_{g}^{2}}{3}
$$

where the lattice speed and the phonon group velocity are related as: $c=2 v_{g} / 3$. Therefore, the Fourier law is recovered through the Chapman-Enskog expansion solution of phonon lattice Boltzmann equation. The three lattice structures and their corresponding lattice speeds are summarized in Table1.

\begin{tabular}{lll}
\hline Lattice type & Discrete lattice velocities & Lattice speed \\
\hline D1Q2 & $( \pm 1,0,0) c$ & $c=\frac{1}{\sqrt{3}} v_{g}$ \\
D2Q8 & $( \pm 1,0,0) c,(0, \pm 1,0) c,( \pm 1, \pm 1,0) c$ & $c=\frac{2}{3} v_{g}$ \\
D3Q14 & $( \pm 1,0,0) c,(0, \pm 1,0) c,(0,0, \pm 1) c,( \pm 1, \pm 1, \pm 1) c$ & $c=\sqrt{\frac{7}{15}} v_{g}$ \\
\hline
\end{tabular}

Table 1: Correlations between lattice speeds and phonon group speed

\section{Results and Discussion}

We analyzed the $\mathcal{Z T}$ of a non-degenerate FSWCNT using the BTE and the phonon LBM. We obtained the carrier thermal conductivity $\left(\kappa_{e}\right)$, and the Peltier coefficient ( $\Pi$ ) based on the analysis of the BTE and simulated the lattice thermal conductivity $\left(\kappa_{\ell}\right)$ based on the phonon LBM. Additionally, we investigated the influence of the doping concentration $\left(n_{o}\right)$ and the overlapping integral for jumps $\left(\Delta_{s}\right.$ and $\left.\Delta_{z}\right)$ on the behavior of the $\mathcal{Z} \mathcal{T}$. The $\mathcal{Z} \mathcal{T}$ was highly nonlinear depending on the doping concentration $n_{o}$, temperature $T$, and real 
overlapping integrals for jumps $\Delta_{s}$ and $\Delta_{z}$. We evaluated numerically the $\mathcal{Z T}$ defined by the relation:

$$
\mathcal{Z} \mathcal{T}=\frac{\sigma \alpha^{2} T}{\kappa_{\ell}+\kappa_{e}}
$$

where $\kappa\left(\kappa_{\ell}+\kappa_{e}\right)$ is characterized by the carrier thermal conductivity $\left(\kappa_{\ell}\right)$ and the lattice thermal conductivity $\left(\kappa_{e}\right)$. The latter plays more important role in the thermal conducativity of FSWCNT. In general, the power factor becomes the main determining parameter in enhancing the $\mathcal{Z T}$ when the thermal conductivity does not vary much.

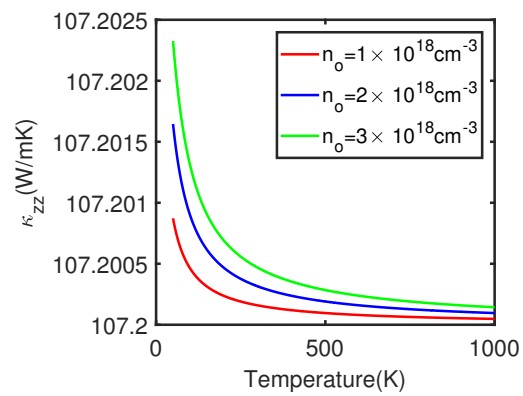

(a)

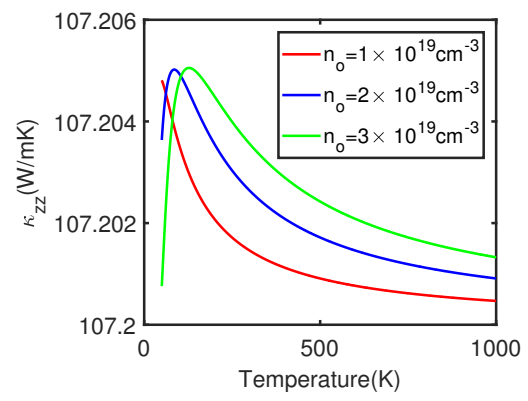

(b)

Figure 2: Dependence of thermal conductivity $\left(\kappa_{z z}\right)$ on temperature $(T)$ with $\Delta_{s}=0.020 \mathrm{eV}$ and $\Delta_{z}=0.013 \mathrm{eV}$ for (a) $n_{o}=1 \times 10^{18} \mathrm{~cm}^{-3}, n_{o}=2 \times 10^{18} \mathrm{~cm}^{-3}$ and $n_{o}=3 \times 10^{18} \mathrm{~cm}^{-3}$ (b) $n_{o}=1 \times 10^{19} \mathrm{~cm}^{-3}, n_{o}=2 \times 10^{19} \mathrm{~cm}^{-3}$ and $n_{o}=3 \times 10^{19} \mathrm{~cm}^{-3}$.

We display in Fig.2 the thermal conductivity $\left(\kappa_{z z}\right)$ dependence on temperature for different doping concentrations, $n_{o}$. When the doping concentration is in the range $10^{18} \mathrm{~cm}^{-3}$, the thermal conductivity is dominated by boundary and phonon-phonon scattering in the high temperature region (Fig.2(a)). The scattering of phonons by impurities (doping), indicates a larger reduction in the thermal conductivity for low doping concentrations for materials like FSWCNT with greater mass and radius differences between the foreign $(F)$ and host atoms $(C)$. This explains the stronger reduction in the thermal conductivity of FSWCNT as observed in Fig.2(a). The strength of the phonon-impurity scattering rate diminishes at lower temperatures. The thermal conductivities of the FSWCNT at low doping concentrations is low, indicating that the phonon-boundary scattering and Umklampp processes dominates over the phonon-impurity scat- 
tering at high temperatures [37-41]. Increasing the doping concentration to the range $\approx 10^{19} \mathrm{~cm}^{-3}$, the thermal conductivity start to show linear behavior in the low temperature regions where scattering is dominated by both phononimperfection and phonon-boundary scattering as shown in Fig.2(b). Phononboundary scattering also contributes significantly to the reduction in the thermal conductivity of the FSWCNT regardless of the level of doping concentration. The peak values of the conductivities for the FSWCNT is determined by the level of impurities in the FSWCNT which is marginal and shift towards high temperatures as the doping concentration increases. However, in doping levels of $10^{19} \mathrm{~cm}^{-3}$ the peak values of the thermal conductivity remains the same. It shows no increase at all as the doping concentration increases, but it is a little bit higher at room temperature for $10^{19} \mathrm{~cm}^{-3}$ than $10^{18} \mathrm{~cm}^{-3}$. This slight increase in the former is due to the increase in doping level as the FSWCNT starts switching from non-degenerate to degenerate character (Fig.2(b)).

In simulating the lattice component of the thermal conductivity $\left(\kappa_{\ell}\right)$ dependence on temperature, we used the phonon LBM. Both thermally excited transverse and longitudinal phonons that interact through normal $(N)$, and Umklapp $(U)$ processes were considered in the simulation. The collision terms were approximated to the relaxation time model with two constants, $\tau_{U}$ and $\tau_{N}$ representing the time(s) required for $U$ and $N$-processes to relax the distribution of phonons to their corresponding equilibrium distributions. Treatments of isothermal, isoflux, adiabatic boundary conditions and periodic temperature gradient boundary conditions were considered during the simulation. Figure (Fig.2(b)) shows specifically a linear temperature dependence at low $T$ which is consistent with one-dimensional band-structure of FSWCNT, with linear acoustic bands contributing to the thermal transport at low temperatures and optical subbands at higher temperatures. The peak of the thermal conductivity for the FSWCNT occurs near $100 \mathrm{~K}$ which separates the low temperature region (where phonon scattering is dominated by imperfections and surfaces), and high temperature regions (where phonon-phonon scattering is dominant). The phonon-phonon (or three-phonon) processes in the FSWCNT are anharmonic effects caused by 
third-order terms in the lattice potential energy. The scattering is a result of the presence of one phonon that causes a periodic elastic strain which through anharmonic interactions modulates in space and time, the elastic constant of the crystal. A second phonon perceives the modulation of the elastic constant and thereafter is scattered to produce a third phonon. The lattice thermal conductivity obtained for FSWCNT is significantly lower than that of pristine samples (SWCNTs) due to the much stronger reduction of phonon mean free path by boundary scattering. A D1Q2 simulation executed for a 15, 000 time-step calculated the lattice thermal conductivity for the FSWCNT to be $107.2 \mathrm{~W} / \mathrm{mK}$ at $300 \mathrm{~K}$ for doping level of $10^{19} \mathrm{~cm}^{-3}$. It is observed from Fig. 2 that, the electronic contribution is minimal and negligible since most of the heat is carried by the phonons. A ratio of the lattice (phonon) to the electronic contribution evaluated shows that, the lattice contribution is about 5 -orders $\left(10^{5}\right)$ of magnitude higher than its electronic component.

The dependence of the axial thermal conductivity $\left(\kappa_{z z}\right)$ on temperature for various values of $\Delta_{z}$ when $\Delta_{s}$ is fixed are presented in Fig.3. It is observed that the thermal conductivity is strongly dominated by the lattice contribution $\left(\kappa_{\ell}\right)$ since FSWCNT is a non-degenerate semiconductor. In the presence of $\nabla T$, the thermal energy is propagated by means of wave packets consisting of various normal modes. The phonon scattering processes included in the Debye approximation for the phonon LBM are resistive and thus, constitute $U$-processes. A strong dependence of the axial thermal conductivity on low temperature $U$-processes $(\exp (\Theta / T)$ ), (where the phonon relaxation time is determined by boundary scattering) and high temperature $U$-processes $\left(T^{-1}\right)$, (where phonon-phonon scatterings are dominant in determining the phonon relaxation time) for $\Delta_{z}=0.013,0.014$ and $0.015 \mathrm{eV}$ are observed (see Fig.3(a)). The $U$-process is observed to be very strong which signifies a strong phononphonon interactions. The total phonon momenta are not conserved during this interaction (phonon-phonon) because the $U$-processes tend to restore nonequilibrium phonon distribution to equilibrium in the FSWCNT and thus, give rise to thermal resistance [42]. However, the $N$-process is observed to be non- 


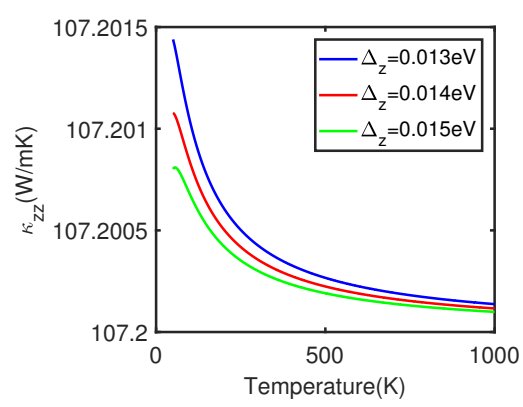

(a)

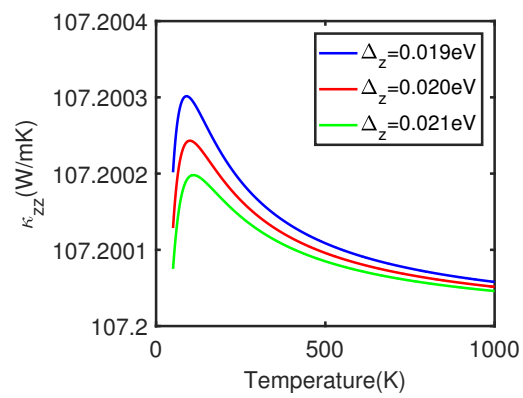

(c)

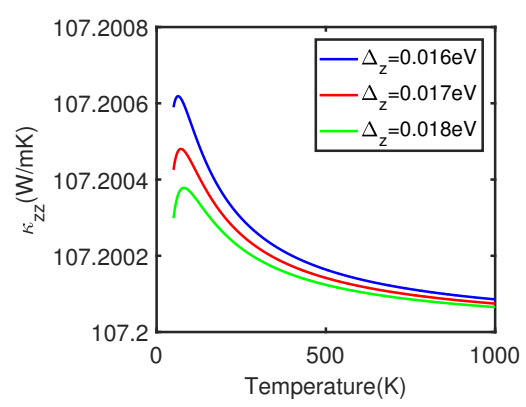

(b)

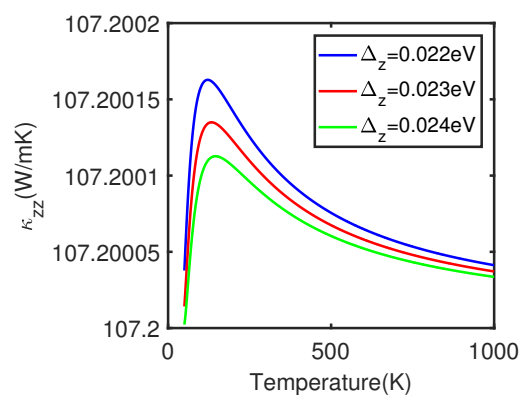

(d)

Figure 3: Dependence of thermal conductivity $\left(\kappa_{z z}\right)$ on temperature $(T)$ with $\Delta_{s}=0.015 \mathrm{eV}$ for (a) $\Delta_{z}=0.013 \mathrm{eV}, \Delta_{z}=0.014 \mathrm{eV}$ and $\Delta_{z}=0.015 \mathrm{eV}$ (b) $\Delta_{z}=0.016 \mathrm{eV}, \Delta_{z}=0.017 \mathrm{eV}$ and $\Delta_{z}=0.018 \mathrm{eV}$ (c) $\Delta_{z}=0.019 \mathrm{eV}, \Delta_{z}=0.020 \mathrm{eV}$ and $\Delta_{z}=0.021 \mathrm{eV}$ (d) $\Delta_{z}=0.022 \mathrm{eV}$, $\Delta_{z}=0.023 \mathrm{eV}$ and $\Delta_{z}=0.024 \mathrm{eV}$.

resistive and conserves the total crystal-momentum but does not contribute to the thermal resistance and yet, has a profound influence on the lattice contributions $\left(\kappa_{\ell}\right)$. These $N$-processes have adverse effect of transferring energy between different phonon modes, thus preventing large deviations from the equilibrium distribution. For $\Delta_{z}=0.016,0.017$ and $0.018 \mathrm{eV}$ the dependence of $\kappa_{z z}$ varies $T^{2}$ as observed in Fig.3(b). Further increase in $\Delta_{z}$ from $\Delta_{z}=0.019-0.024 \mathrm{eV}$ reveals a linear conductivity as shown in Fig.3(c) and Fig.3(d). This emanates from phonon-imperfections and phonon-boundary scattering that arise from the doping processes and the increase in doping concentration $\left(\kappa \approx T^{3}\right)$.

We have previously reported the dependence of the axial thermopower and power factor on temperature for $\Delta_{s}, \Delta_{z}, E_{o}$ and $n_{o}$. The thermopower decreased rapidly with increasing temperature and at high temperatures, approached 
a constant value (hyperbolic in nature). This behavior has been observed in semiconducting tubes where $\alpha_{z z} \approx T^{-1}$ [6-8,43-45]. The FSWCNT behaved purely as a $p$-type semiconductor initially but switches to $n$-type behavior at higher temperatures as the $\Delta_{s}$ increases [8].

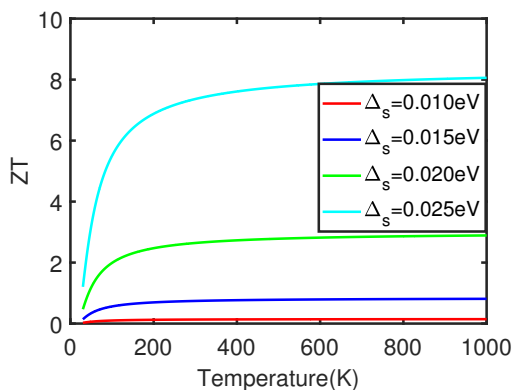

(a)

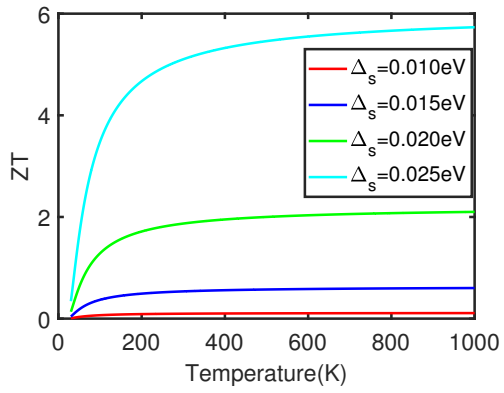

(c)

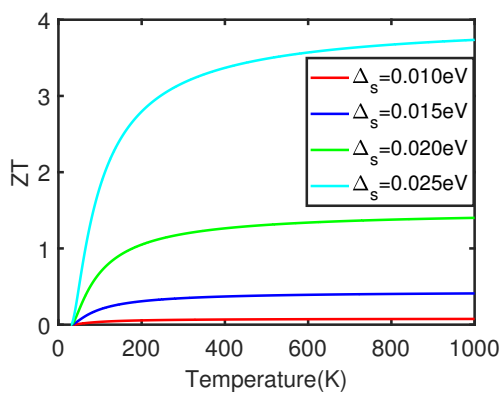

(e)

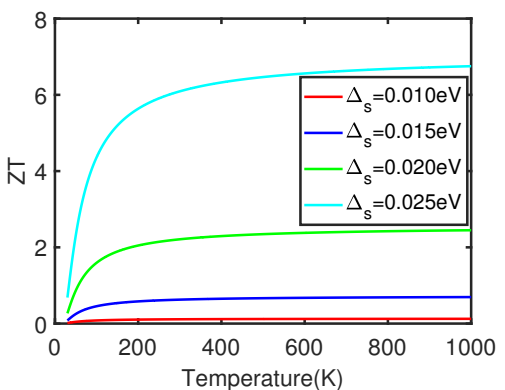

(b)

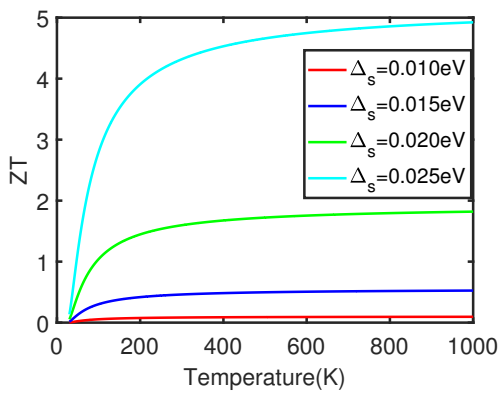

(d)

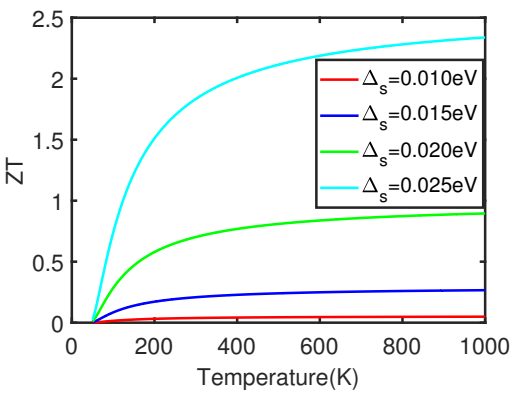

(f)

Figure 4: Dependence of thermoelecric figure of merit $(\mathcal{Z T})$ on temperature $T$ for $n_{o}=$ $10^{19} \mathrm{~cm}^{-3}$ and $E_{z}=10^{2} \mathrm{~V} / \mathrm{m}$ for different values of $\Delta_{s}$ with (a) $\Delta_{z}=0.015 \mathrm{eV}$ (b) $\Delta_{z}=$ $0.016 \mathrm{eV}$ (c) $\Delta_{z}=0.017 \mathrm{eV}$, (d) $\Delta_{z}=0.018 \mathrm{eV}$ (e) $\Delta_{z}=0.020 \mathrm{eV}$ and (f) $\Delta_{z}=0.024 \mathrm{eV}$.

The $\mathcal{Z} \mathcal{T}$ dependence on temperature for various values of $\Delta_{s}$ when $\Delta_{z}$ is fixed 
is displayed in Fig.4. The $\mathcal{Z} \mathcal{T}$ rises linearly and saturates as the temperature increases above $100 \mathrm{~K}$ for $\Delta_{z}=0.015 \mathrm{eV}$ when $\Delta_{s}$ is varied. The saturation of the $\mathcal{Z} \mathcal{T}$ is due to the maximum velocity (saturation velocity) the carriers in FSWCNT attains in the presence of high fields. The FSWCNT carriers move at an average drift velocity proportional to the strength of $\nabla T$ with time. The higher the mobility of the FSWCNT carriers, the higher the drift velocity and consequently higher intraminiband current values for the given field strength. A limit is reached around $100 K$ where further high field values do not allow the FSWCNT carriers to move any faster, having reached its saturation velocity, due to several mechanisms that eventually limit the movement of the carriers in the FSWCNT. As the applied field $\nabla T$ increases from this point $(T=100 K)$, the FSWCNT carrier velocity no longer increases because the carriers lose energy through increased levels of interaction with the lattice, by emitting phonons and even photons since the carrier energy is large enough to do so. Thus, at high fields (i.e. high $\nabla T$ ) scattering of intraminiband electrons by phonons increases for fixed $\Delta_{z}$ which induces a high-frequency carrier dynamics $[8,23]$ that depend critically on the magnitude of $\nabla T$. The carrier velocity is drifted through the FSWCNT and allowed to perform drifting periodic orbits (in $\mathrm{THz}$ frequencies) $[8,23]$ leading to a resonant enhancement of $Z T$ which saturates (i.e. $\nabla T \approx 0$ ). In Fig.4(a), we observe the $\mathcal{Z} \mathcal{T}$ value to be very high i.e $\mathcal{Z} \mathcal{T}>6$. This value of $\mathcal{Z} \mathcal{T}$ starts saturating gradually as the value of $\Delta_{z}$ increases from $0.015 \mathrm{eV}$ to $0.025 \mathrm{eV}$ (Fig.4(a) and Fig.4(b)). In this case, the electron scattering along the axial direction decreases for increasing values of $\Delta_{s}$ when $\Delta_{z}$ is fixed and low. The number of intraminiband electrons scattered by the lattice vibrations are less and so a greater number of the intraminiband electrons contributes to the electrical conductivity $(\sigma)$ and consequently the power factor $\left(\alpha^{2} \sigma\right)$. Thus, $\alpha^{2} \sigma T \gg \kappa$ and so the $\mathcal{Z} \mathcal{T}$ keeps rising as high as $\mathcal{Z} \mathcal{T}>6$. However, the electron scattering along the axial direction start increasing for increasing values of $\Delta_{s}$ when $\Delta_{z}$ is fixed. This means the number of intraminiband electrons scattered by the lattice vibrations are more and so a lesser number of intraminiband electrons contributes to the electrical conductivity $(\sigma)$ and consequently the 
power factor $\left(\alpha^{2} \sigma\right)$ [8]. Thus, $\alpha^{2} \sigma T \ll \kappa$ and so the $\mathcal{Z} \mathcal{T}$ keeps decreasing, $\mathcal{Z} \mathcal{T}<6$ (Fig.4(c)-Fig.4(f))

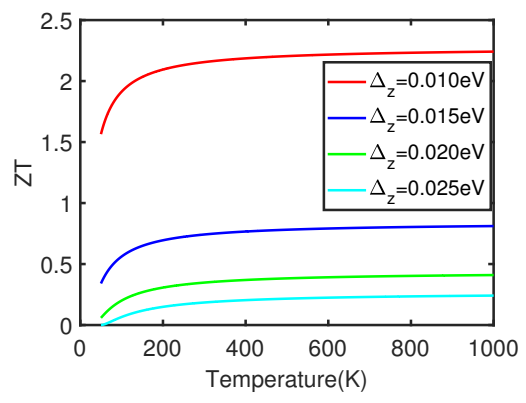

(a)

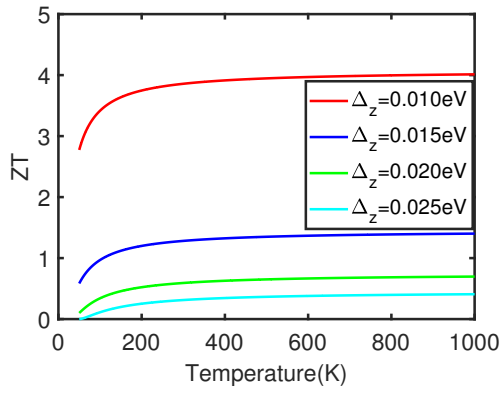

(c)

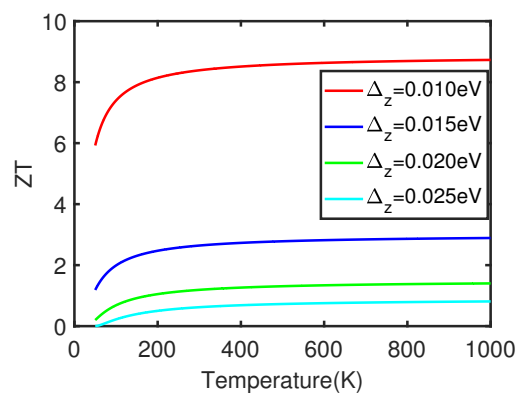

(e)

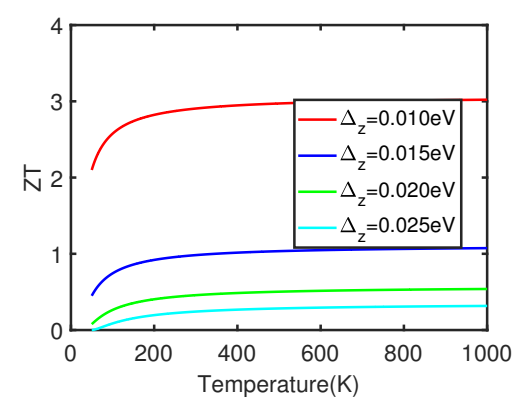

(b)

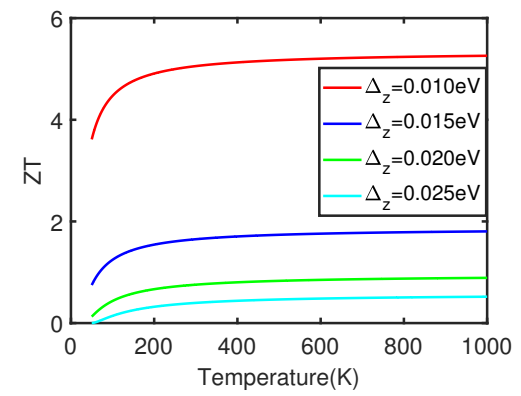

(d)

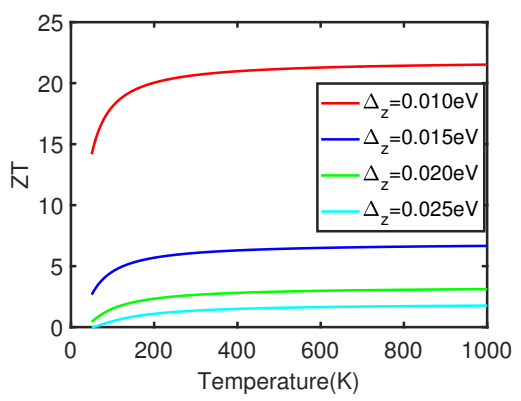

(f)

Figure 5: Dependence of thermoelecric figure of merit $(\mathcal{Z T})$ on temperature $T$ for $n_{o}=$ $10^{19} \mathrm{~cm}^{-3}$ and $E_{z}=10^{2} \mathrm{~V} / \mathrm{m}$ for different values of $\Delta_{s}$ with (a) $\Delta_{s}=0.015 \mathrm{eV}$ (b) $\Delta_{s}=$ $0.016 \mathrm{eV}$ (c) $\Delta_{s}=0.017 \mathrm{eV}$, (d) $\Delta_{s}=0.018 \mathrm{eV}$ (e) $\Delta_{s}=0.020 \mathrm{eV}$ and (f) $\Delta_{s}=0.024 \mathrm{eV}$.

In Fig. 5 we display the dependence of $\mathcal{Z} \mathcal{T}$ for various values of $\Delta_{z}$ when $\Delta_{s}$ is fixed. The $\mathcal{Z} \mathcal{T}$ exhibit similar behavior as discussed in Fig.4. The $\mathcal{Z} \mathcal{T}$ in Fig. 5 a is very low, but start to increase gradually as the value of $\Delta_{s}$ increases from 
$0.015 \mathrm{eV}$ to $0.024 \mathrm{eV}$ (Fig.5(a)-Fig.5(f)). Thus, $\alpha^{2} \sigma T \gg \kappa$ and so the $\mathcal{Z} \mathcal{T}$ keeps rising (i.e. $\mathcal{Z} \mathcal{T}>20$ ).

\section{Conclusion}

The thermoelectric figure of merit $(\mathcal{Z} \mathcal{T})$ of a non-degenerate FSWCNT was investigated using a tractable analytical approach and the phonon LBM. The FSWCNT parameters $\Delta_{s}, \Delta_{z}$, and $n_{o}$ were found to influence the $\mathcal{Z} \mathcal{T}$ strongly. At high fields (high $\nabla T$ ), there is an increase in scattering for fixed $\Delta_{z}$ which induces a high-frequency carrier dynamics that depend critically on the magnitude of $\nabla T$. The carrier velocity is drifted through the FSWCNT and allowed to perform drifting periodic orbits (in $\mathrm{THz}$ frequencies) leading to a resonant enhancement of $\mathcal{Z} \mathcal{T}$ which saturates at high temperatures (i.e. $\nabla T \approx 0$ ). Optimizing $\Delta_{s}, \Delta_{z}, T$ and $n_{o}$ can yield a $\mathcal{Z} \mathcal{T}$ greater $20(\mathcal{Z} \mathcal{T}>20)$ for high $\Delta_{s}$. Interestingly, varying the $n_{o}, \Delta_{s}$ and $\Delta_{z}$ can be used to tune the FSWCNT to operate at higher temperatures. However, based on the $\mathcal{Z} \mathcal{T}$ obtained at weak electric field, we propose FSWCNT as a potential candidate for thermoelectric application. Furthermore, contemporary techniques can be employed to reduce $\kappa_{\ell}$

\section{References}

[1] G. S. Nolas, J. Sharp, and H. J. Goldsmid, Thermoelectrics: Basic Principles and New Materials Developments (Springer, Berlin, 2001).

[2] Rowe, D. M., Gao Min, and L. Kuznestsov. "Electrical resistivity and Seebeck coefficient of hot-pressed $\mathrm{YbAl}_{3}$ over the temperature range 150700K." Philosophical magazine letters 77, no. 2 (1998): 105-108.

[3] Hicks, L. D., and Mildred S. Dresselhaus. "Effect of quantum-well structures on the thermoelectric figure of merit." Physical Review B 47, no. 19 (1993): 12727.

[4] Rowe D.M. and Min G. 1994, Proceedings of the 13th International Conference on Thermoelectrics, Kansas, p336 
[5] Balandin, Alexander, and Kang L. Wang. "Effect of phonon confinement on the thermoelectric figure of merit of quantum wells." Journal of Applied Physics 84, no. 11 (1998): 6149-6153.

[6] Slepyan, Gregory Ya, Sergey A. Maksimenko, Akhlesh Lakhtakia, Oleg M. Yevtushenko, and Anton V. Gusakov. "Electronic and electromagnetic properties of nanotubes." Physical Review B 57, no. 16 (1998): 9485.

[7] Mensah, Samuel Yeboah, F. K. A. Allotey, N. G. Mensah, and G. Nkrumah. "Differential thermopower of a CNT chiral carbon nanotube." Journal of Physics: Condensed Matter 13, no. 24 (2001): 5653.

[8] Sakyi-Arthur, D., Mensah, S. Y., K.W. Adu, Mensah, N. G., Dompreh, K. A., and Edziah, R. Tunable power factor of fluorine-doped carbon nanotube. J. Appl. Phys. 128, 244301 (2020). doi: 10.1063/5.0031326

[9] Tan, X., Liu, H., Wen, Y., Lv, H., Pan, L., Shi, J., and Tang, X. (2012). Optimizing the thermoelectric performance of zigzag and chiral carbon nanotubes. Nanoscale research letters, 7(1), 1-7.

[10] H. Lee, D. Vashaee, D. Z. Wang,3 M.S. Dresselhaus, Z. F. Ren, G Chen., Effects of nanoscale porosity on thermoelectric properties of SiGe, JOURNAL OF APPLIED PHYSICS 107, 094308 (2010).doi:10.1063/1.3388076

[11] Zhao, L. D., Lo, S. H., Zhang, Y., Sun, H., Tan, G., Uher, C., and Kanatzidis, M. G. (2014). Ultralow thermal conductivity and high thermoelectric figure of merit in SnSe crystals. Nature, 508(7496), 373-377.

[12] Liao, B., Qiu, B., Zhou, J., Huberman, S., Esfarjani, K., and Chen, G. (2015). Significant reduction of lattice thermal conductivity by the electronphonon interaction in silicon with high carrier concentrations: A firstprinciples study. Physical review letters, 114(11), 115901.

[13] Li, J. B., Wang, J., Li, J. F., Li, Y., Yang, H., Yu, H. Y., and Miao, L. (2018). Broadening the temperature range for high thermoelectric perfor- 
mance of bulk polycrystalline strontium titanate by controlling the electronic transport properties. Journal of Materials Chemistry C, 6(28), 75947603.

[14] Venkatasubramanian, R., Siivola, E., Colpitts, T., and O'quinn, B. (2001). Thin-film thermoelectric devices with high room-temperature figures of merit. Nature, 413(6856), 597-602.

[15] Lin, Y. M., Sun, X., and Dresselhaus, M. S. (2000). Theoretical investigation of thermoelectric transport properties of cylindrical Bi nanowires. Physical Review B, 62(7), 4610.

[16] M.S. Dresselhaus, Y.M. Lin, T. Koga, S.B. Cronin, O. Rabin, M.R. Black, and G. Dresselhaus, in Semiconductors and Semimetals: Recent Trends in Thermoelectric Materials Research III, edited by T.M. Tritt (Academic, San Diego, CA, 2001), Chap. 1.

[17] Harman, T. C., Taylor, P. J., Walsh, M. P., and LaForge, B. E. (2002). Quantum dot superlattice thermoelectric materials and devices. science, 297(5590), 2229-2232.

[18] Piraux, L., George, J. M., Despres, J. F., Leroy, C., Ferain, E., Legras, R., and Fert, A. (1994). Giant magnetoresistance in magnetic multilayered nanowires. Applied Physics Letters, 65(19), 2484-2486.

[19] Balandin, A. A., and Lazarenkova, O. L. (2003). Mechanism for thermoelectric figure-of-merit enhancement in regimented quantum dot superlattices. Applied Physics Letters, 82(3), 415-417.

[20] Klemens, P. G. (1966). Anharmonic decay of optical phonons. Physical Review, 148(2), 845 .

[21] Sakyi-Arthur, D., S. Y. Mensah, N. G. Mensah, K. A. Dompreh, and R. Edziah. Absorption of Acoustic Phonons in Fluorinated Carbon Nanotube with Non-parabolic, Double Periodic Band. Phonons in Low Dimensional 
Structures (pp.129-142). InTech. doi: doi.org/10.5772/intechopen.78231 (2018)

[22] Sakyi-Arthur, D., Mensah, S.Y., Adu, K.W., Dompreh, K.A., Edziah, R.Mensah, N.G., and Jebuni-Adanu, C. (2020). Induced Halllike current by Acoustic Phonons in Semiconductor Fluorinated Carbon Nanotube .World Journal of Condensed Matter Physics, 10, 7187.https://doi.org/10.4236/wjcmp.2020. 102005

[23] Sakyi-Arthur, D., Mensah, S.Y., Adu, K.W., Dompreh, K.A., Edziah, R. and Mensah, N.G. (2020). Acoustoelectric Effect in Fluorinated Carbon Nanotube in the Absence of External Electric Field. World Journal of Condensed Matter Physics, 10, 1-11.https://doi.org/10.4236/wjcmp.2020. 101001

[24] Sakyi-Arthur, D., Mensah, S.Y., Adu, K.W., Dompreh, K.A., Edziah, R., Mensah, N.G., and Jebuni-Adanu, C. (2020). Semiconductor Fluorinated Carbon Nanotube as a Low Voltage Current Amplifier Acoustic Device. World Journal of Condensed Matter Physics, 10, 12-26.https://doi.org/ 10.4236/wjcmp.2020.101002

[25] Sadykov, N. R., E. Yu Kocherga, and P. N. Dyachkov. "Nonlinear current in modified nanotubes with exposure to alternating and constant electric fields." Russian Journal of Inorganic Chemistry 58, no. 8 (2013): 951-955.

[26] Y. Guo, M. Wang, Lattice Boltzmann modeling of phonon transport. Journal of Computational Physics. doi.org/10.1016/j.jcp.2016.03.041

[27] S. Succi, The Lattice Boltzmann Equation for Fluid Dynamics and Beyond, Clarendon Press, Oxford, 2001.

[28] Z.L. Guo, C. Shu, Lattice Boltzmann Method and Its Applications in Engineering, World Scientific Publishing, Singapore, 2013.

[29] H.D. Chen, S.Y. Chen, W.H. Matthaeus, Recovery of the NavierStokes equations using a latticegas Boltzmann method, Phys. Rev. A 45 (1992) R5339R5342. 
[30] X.Y. He, L.S. Luo, Theory of the lattice Boltzmann method: from the Boltzmann equation to the lattice Boltzmann equation, Phys. Rev. E 56 (1997) 68116817.

[31] S.Y. Chen, G.D. Doolen, Lattice Boltzmann method for fluid flows, Annu. Rev. Fluid Mech. 30 (1998) 329364.

[32] X.W. Shan, G. Doolen, Diffusion in a multicomponent lattice Boltzmann equation model, Phys. Rev. E 54 (1996) 36143620.

[33] J.K. Wang, M. Wang, Z.X. Li, Lattice PoissonBoltzmann simulations of electro-osmotic flows in microchannels, J. Colloid Interface Sci. 296 (2006) 729736 .

[34] J. Wang, M. Wang, Z. Li, A lattice Boltzmann algorithm for fluidsolid conjugate heat transfer, Int. J. Therm. Sci. 46 (2007) 228234.

[35] J. Zhang, G. Yan, X. Shi, Lattice Boltzmann model for wave propagation, Phys. Rev. E 80 (2009) 026706.

[36] Y. Ma, S.K. Dong, H.P. Tan, Lattice Boltzmann method for onedimensional radiation transfer, Phys. Rev. E 84 (2011) 016704.

[37] Carey, V. P., Chen, G., Grigoropoulos, C., Kaviany, M., and Majumdar, A. (2008). A review of heat transfer physics. Nanoscale and Microscale Thermophysical Engineering, 12(1), 1-60.

[38] Ziman, J. M. (1956). XVII. The effect of free electrons on lattice conduction. Philosophical Magazine, 1(2), 191-198.

[39] Ziman, J. M. (1957). The effect of free electrons on lattice conduction. Philosophical Magazine, 2(14), 292-292.

[40] Kosarev, V. (1971). Scattering of phonons by carriers in the field of charged impurities. SOVIET PHYSICS JETP, 33(4). 
[41] Radhakrishnan, V., Sharma, P. C., and Singh, M. (1980). Electron-phonon interaction inP-doped silicon at low temperatures. Zeitschrift fr Physik B Condensed Matter, 39(1), 15-19.

[42] Tritt, Terry M., ed. Thermal conductivity: theory, properties, and applications. Springer Science \& Business Media, 2005.

[43] Mensah, S. Y., and G. K. Kangah. "The thermoelectric effect in a semiconductor superlattice in a non-quantized electric field." Journal of Physics: Condensed Matter 4, no. 3 (1992): 919.

[44] Mensah, S.Y, F. K. A. Allotey, N. G. Mensah, and G. Nkrumah. "Differential thermopower of a CNT chiral carbon nanotube." Journal of Physics: Condensed Matter 13, no. 24 (2001): 5653.

[45] Sumanasekera, G. U., Adu, C. K. W., Fang, S., and Eklund, P. C. (2000). Effects of gas adsorption and collisions on electrical transport in singlewalled carbon nanotubes. Physical Review Letters, 85(5), 1096. 